\title{
Properties of baryon resonances from a multichannel partial wave analysis
}

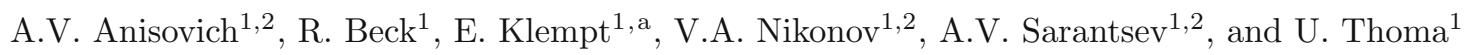 \\ 1 Helmholtz-Institut für Strahlen- und Kernphysik, Universität Bonn, Germany \\ 2 Petersburg Nuclear Physics Institute, Gatchina, Russia
}

Received: 20 December 2011 / Revised: 18 January 2012

Published online: 13 February 2012 - C Società Italiana di Fisica / Springer-Verlag 2012

Communicated by U.-G. Meißner

\begin{abstract}
Properties of nucleon and $\Delta$ resonances are derived from a multichannel partial wave analysis of pion and photo-induced reactions off protons. This paper summarizes the latest results on masses, widths, and decay properties of nucleon and $\Delta$ resonances.
\end{abstract}

\section{Introduction}

Existence and properties of most $N$ and $\Delta$ resonances listed in the Review of Particle Properties [1] were derived from partial wave analyses of $\pi N$ elastic and charge exchange scattering data [2-5]. Additional information on their decay modes was obtained from inelastic reactions, from $\pi N \rightarrow N \eta, \Lambda K, \Sigma K$ and from an isobar model study of $\pi N \rightarrow N \pi \pi$; photoproduction experiments provided information on the photo-coupling. The most recent analysis [5] — based on a larger data set and on very precise data from meson factories - found no evidence for the existence of 16 of the $32 N$ and $\Delta$ resonances below $2.2 \mathrm{GeV}$ listed in the Baryon Particle Tables. Obviously, the existing database was not sufficient to extract a reliable spectrum of $N$ and $\Delta$ resonances from pion-induced reactions alone.

In the last years, an impressive amount of photoinduced reactions has been studied at ELSA, GRAAL, Jlab, MAMI, and SPring-8, and the situation has changed significantly. High-statistics data are available not only on differential cross-sections but also on many polarization observables. In particular, reactions like $\gamma p \rightarrow p \pi^{0}, n \pi^{+}$, $p \eta, p \pi^{0} \pi^{0}, p \pi^{+} \pi^{-}, p \pi^{0} \eta, \Lambda K^{+}, \Sigma^{0} K^{+}$, and $\Sigma^{+} K_{s}^{0}$ have been studied, some of them in great detail.

In this paper, we give a brief account of the results of the Bonn-Gatchina (BnGa) multichannel partial wave analysis. Main results have been reported before [6-9]. We found two classes of solutions, called BnGa2011-01 and BnGa2011-02, which differ in the number and properties of some positive-parity nucleon resonances at masses above $1.9 \mathrm{GeV}$. The emphasis of the papers $[7,9]$ was on a discussion of the alternative solutions, on the new resonances found in the analysis, and on their physics interpretation. In [6], amplitudes for pion photoproduction off protons were presented, and, in [8], the focus was to explore

\footnotetext{
a e-mail: klempt@hiskp.uni-bonn.de
}

possible interpretations of a narrow structure in the $N \eta$ mass distribution. The emphasis here is to provide complete information on resonances, their masses and widths, their helicity amplitudes, and their decay properties. Included here are new results on $\gamma p \rightarrow p 2 \pi^{0}$ for photons in the energy range up to $3 \mathrm{GeV}[10]$, and on the polarization observables $I^{s}$ and $I^{c}$ in the reactions $\gamma p \rightarrow p 2 \pi^{0}[11]$ and $\gamma p \rightarrow p \pi^{0} \eta[12]$ which characterize correlations between a linear photon polarization and the direction of outgoing single particles. These new data improve the knowledge of decay modes of baryon resonances into $p \pi^{0} \pi^{0}$. The main results are unchanged, hence we call the new solution BnGa2011-02a. Compared to our previous publications, the error analysis has been improved by storing several acceptable solutions and by calculating (instead of estimating) properties and errors from the distribution of all quantities. Hence the results supersede those of $[7,9]$.

\section{Data used in the partial wave analysis}

Tables 1-6 give an updated list of the pion- and photoinduced reactions used in the coupled-channel analysis presented here. The data comprise most of the important reactions including multiparticle final states. Resonances with sizable coupling constants to $\pi N$ and $\gamma N$ are thus unlikely to escape the fits even though further single and double polarization experiments are certainly needed to unambiguously constrain the contributing amplitudes. The tables list the reaction, the observables and references to the data, the number of data points, the weight with which the data are used in the fits, and the $\chi^{2}$ per data point of our final solution BnGa2011-02a, a solution which is derived from BnGa2011-02 but includes the data from [10-12]. We use the $\pi N$ elastic amplitudes from [5] since they do not provide any bias for additional 
Table 1. Fit to the real and imaginary part of elastic $\pi N$ amplitudes and $\chi^{2}$ contributions for the solution BG2011-02a. The elastic scattering data are fitted jointly with a larger number of further data in a coupled-channel approach. We use the amplitudes from [5] not to bias the analysis towards more resonances.

\begin{tabular}{ccccc}
\hline \hline$\pi N \rightarrow \pi N$ & Wave & $N_{\text {data }}$ & $w_{i}$ & $\chi_{i}^{2} / N_{\text {data }}$ \\
\hline$[5]$ & $S_{11}$ & 112 & 30 & 2.11 \\
& $S_{31}$ & 112 & 20 & 2.19 \\
$P_{11}$ & 112 & 70 & 1.70 \\
& $P_{31}$ & 104 & 20 & 3.74 \\
$P_{13}$ & 112 & 25 & 1.39 \\
$P_{33}$ & 120 & 15 & 2.77 \\
$D_{13}$ & 108 & 10 & 2.21 \\
$D_{33}$ & 108 & 12 & 3.08 \\
$D_{15}$ & 104 & 20 & 2.29 \\
& $F_{15}$ & 88 & 30 & 1.87 \\
$F_{35}$ & 62 & 20 & 1.64 \\
& $F_{37}$ & 72 & 10 & 2.76 \\
& $F_{17}$ & 82 & 30 & 1.99 \\
& $G_{17}$ & 102 & 15 & 2.31 \\
& $G_{19}$ & 74 & 15 & 2.82 \\
& $H_{19}$ & 86 & 15 & 2.56 \\
\hline \hline
\end{tabular}

Table 2. Pion-induced reactions fitted in the coupled-channel analysis and $\chi^{2}$ contributions for the solution BG2011-02a.

\begin{tabular}{ccccc}
\hline \hline$\pi^{-} p \rightarrow \eta n$ & Observ. & $N_{\text {data }}$ & $w_{i}$ & $\chi_{i}^{2} / N_{\text {data }}$ \\
\hline$[14]$ & $\mathrm{d} \sigma / \mathrm{d} \Omega$ & 70 & 20 & 1.47 \\
{$[15]$} & $\mathrm{d} \sigma / \mathrm{d} \Omega$ & 84 & 30 & 2.98 \\
\hline \hline$\pi^{-} p \rightarrow K^{0} \Lambda$ & Observ. & $N_{\text {data }}$ & $w_{i}$ & $\chi_{i}^{2} / N_{\text {data }}$ \\
\hline$[16]$ & $\mathrm{d} \sigma / \mathrm{d} \Omega$ & 300 & 30 & 0.90 \\
{$[17,18]$} & $\mathrm{d} \sigma / \mathrm{d} \Omega$ & 298 & 30 & 2.30 \\
{$[17,18]$} & $P$ & 355 & 30 & 1.77 \\
{$[19]$} & $\beta$ & 72 & 70 & 1.06 \\
\hline \hline$\pi^{+} p \rightarrow K^{+} \Sigma^{+}$ & Observ. & $N_{\text {data }}$ & $w_{i}$ & $\chi_{i}^{2} / N_{\text {data }}$ \\
\hline$[20-24]$ & $\mathrm{d} \sigma / \mathrm{d} \Omega$ & 728 & 35 & 1.46 \\
{$[20-25]$} & $P$ & 351 & 30 & 1.57 \\
{$[26]$} & $\beta$ & 7 & 600 & 2.04 \\
\hline \hline$\pi^{-} p \rightarrow K^{0} \Sigma^{0}$ & Observ. & $N_{\text {data }}$ & $w_{i}$ & $\chi_{i}^{2} / N_{\text {data }}$ \\
\hline$[27]$ & $\mathrm{d} \sigma / \mathrm{d} \Omega$ & 259 & 30 & 0.98 \\
{$[27]$} & $P$ & 95 & 30 & 1.30 \\
\hline \hline
\end{tabular}

Table 3. Observables from $\eta$ photoproduction fitted in the coupled-channel analysis and $\chi^{2}$ contributions for the solution BG2011-02a.

\begin{tabular}{lcccc}
\hline \hline$\gamma p \rightarrow \eta p$ & Observ. & $N_{\text {data }}$ & $w_{i}$ & $\chi_{i}^{2} / N_{\text {data }}$ \\
\hline [110] Crystal Ball @ MAMI & $\mathrm{d} \sigma / \mathrm{d} \Omega$ & 2400 & 2 & 1.30 \\
{$[111]$ CBT } & $\mathrm{d} \sigma / \mathrm{d} \Omega$ & 680 & 40 & 1.39 \\
[112] CB & $\mathrm{d} \sigma / \mathrm{d} \Omega$ & 631 & 20 & 1.74 \\
{$[113]$ GRAAL } & $\Sigma$ & 51 & 10 & 1.81 \\
[114] GRAAL & $\Sigma$ & 150 & 15 & 1.19 \\
[115] CBT & $\Sigma$ & 34 & 20 & 0.82 \\
\hline \hline
\end{tabular}

Table 4. Observables from $\pi$ photoproduction fitted in the coupled-channel analysis and $\chi^{2}$ contributions for the solution BG2011-02a.

\begin{tabular}{lcccc}
\hline \hline$\gamma p \rightarrow \pi^{0} p$ & Observ. & $N_{\text {data }}$ & $w_{i}$ & $\chi_{i}^{2} / N_{\text {data }}$ \\
\hline$[36]$ (TAPS@MAMI) & $\mathrm{d} \sigma / \mathrm{d} \Omega$ & 1692 & 0.8 & 1.61 \\
{$[37,38](\mathrm{GDH} \mathrm{A} 2)$} & $\mathrm{d} \sigma / \mathrm{d} \Omega$ & 164 & 7 & 1.19 \\
{$[39](\mathrm{GRAAL})$} & $\mathrm{d} \sigma / \mathrm{d} \Omega$ & 861 & 2 & 1.56 \\
{$[40,41](\mathrm{CB})$} & $\mathrm{d} \sigma / \mathrm{d} \Omega$ & 1106 & 3.5 & 1.59 \\
{$[42](\mathrm{CLAS})$} & $\mathrm{d} \sigma / \mathrm{d} \Omega$ & 592 & 6 & 1.19 \\
{$[43](\mathrm{CBT})$} & $\mathrm{d} \sigma / \mathrm{d} \Omega$ & 540 & 6 & 2.01 \\
{$[39,44-51]$} & $\Sigma$ & 1492 & 3 & 2.65 \\
{$[52](\mathrm{CBT})$} & $\Sigma$ & 374 & 30 & 1.04 \\
{$[45-47,53-62]$} & $T$ & 389 & 8 & 3.24 \\
{$[45-47,62-66]$} & $P$ & 607 & 3 & 3.14 \\
{$[67,68]$} & $G$ & 75 & 5 & 1.49 \\
{$[67]$} & $H$ & 71 & 5 & 1.22 \\
{$[37,38]$} & $E$ & 140 & 7 & 1.03 \\
{$[65,69]$} & $O_{x^{\prime}}$ & 7 & 10 & 1.14 \\
{$[65,69]$} & $O_{z^{\prime}}$ & 7 & 10 & 0.35 \\
\hline \hline$\gamma p \rightarrow \pi^{+} n$ & $\mathrm{Observ}$. & $N_{\text {data }}$ & $w_{i}$ & $\chi_{i}^{2} / N_{\text {data }}$ \\
\hline$[70-81]$ & $\mathrm{d} \sigma / \mathrm{d} \Omega$ & 1583 & 2 & 1.33 \\
{$[38,82](\mathrm{GDH} \mathrm{A} 2)$} & $\mathrm{d} \sigma / \mathrm{d} \Omega$ & 408 & 14 & 0.69 \\
{$[83](\mathrm{CLAS})$} & $\mathrm{d} \sigma / \mathrm{d} \Omega$ & 484 & 4 & 1.12 \\
{$[51,84-94]$} & $\Sigma$ & 899 & 3 & 3.46 \\
{$[89,90,95-105]$} & $T$ & 661 & 3 & 3.09 \\
{$[89,90,106]$} & $P$ & 252 & 3 & 2.20 \\
{$[68,107,108]$} & $G$ & 86 & 8 & 5.47 \\
{$[107-109]$} & $H$ & 128 & 3 & 3.75 \\
{$[38,82]$} & $E$ & 231 & 14 & 1.52 \\
\hline \hline & & & & \\
\hline
\end{tabular}

Table 5. Reactions leading to 3-body final states included in the event-based likelihood fits; likelihood values for the solution BG2011-02a. CB stands for CB-ELSA; CBT for CBELSA/TAPS.

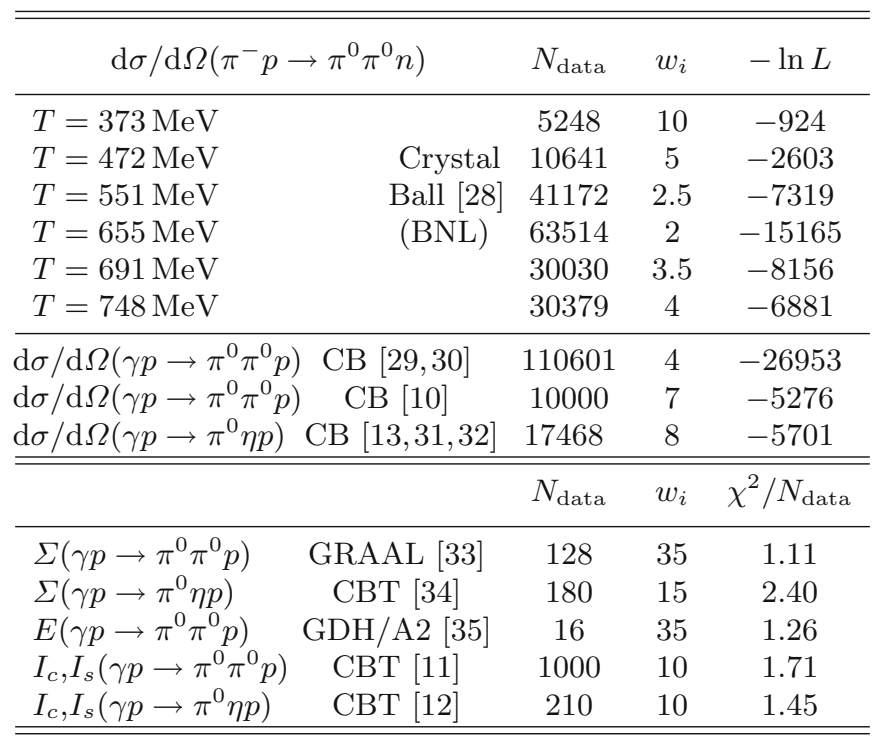

resonances. Multibody final states are fitted in an eventbased likelihood fit. For these reactions, the log likelihood is given (see eq. (18)). Inelastic reactions with polarized photons are included as histograms. The analysis was con- 
Table 6. Hyperon photoproduction observables fitted in the coupled-channel analysis and $\chi^{2}$ contributions for the solution BG2011-02a.

\begin{tabular}{|c|c|c|c|c|}
\hline$\gamma p \rightarrow K^{+} \Lambda$ & Observ. & $N_{\text {data }}$ & $w_{i}$ & $\chi_{i}^{2} / N_{\text {data }}$ \\
\hline [116] CLAS & $\mathrm{d} \sigma / \mathrm{d} \Omega$ & 1320 & 16 & 0.69 \\
\hline [117] LEPS & $\Sigma$ & 45 & 10 & 2.11 \\
\hline [118] GRAAL & $\Sigma$ & 66 & 8 & 2.95 \\
\hline [116] CLAS & $P$ & 1270 & 8 & 1.82 \\
\hline [118] GRAAL & $P$ & 66 & 10 & 0.59 \\
\hline [119] GRAAL & $T$ & 66 & 15 & 1.62 \\
\hline [120] CLAS & $C_{x}$ & 160 & 15 & 1.52 \\
\hline [120] CLAS & $C_{z}$ & 160 & 15 & 1.58 \\
\hline [119] GRAAL & $O_{x^{\prime}}$ & 66 & 12 & 1.95 \\
\hline [119] GRAAL & $O_{z^{\prime}}$ & 66 & 15 & 1.66 \\
\hline$\gamma p \rightarrow K^{+} \Sigma$ & Observ. & $N_{\text {data }}$ & $w_{i}$ & $\chi_{i}^{2} / N_{\text {data }}$ \\
\hline [121] CLAS & $\mathrm{d} \sigma / \mathrm{d} \Omega$ & 1590 & 3 & 1.44 \\
\hline [117] LEPS & $\Sigma$ & 45 & 10 & 1.23 \\
\hline [118] GRAAL & $\Sigma$ & 42 & 10 & 1.99 \\
\hline [121] CLAS & $P$ & 344 & 12 & 2.69 \\
\hline [120] CLAS & $C_{x}$ & 94 & 15 & 1.95 \\
\hline [120] CLAS & $C_{z}$ & 94 & 15 & 1.66 \\
\hline$\gamma p \rightarrow K^{0} \Sigma^{+}$ & Obsv. & $N_{\text {data }}$ & $w_{i}$ & $\chi_{i}^{2} / N_{\text {data }}$ \\
\hline [122] CLAS & $\mathrm{d} \sigma / \mathrm{d} \Omega$ & 48 & 3 & 3.84 \\
\hline [123] SAPHIR & $\mathrm{d} \sigma / \mathrm{d} \Omega$ & 160 & 5 & 1.91 \\
\hline [124] CBT & $\mathrm{d} \sigma / \mathrm{d} \Omega$ & 72 & 10 & 0.76 \\
\hline [125] CBT & $\mathrm{d} \sigma / \mathrm{d} \Omega$ & 72 & 40 & 0.62 \\
\hline [124] CBT & $P$ & 72 & 15 & 0.90 \\
\hline [125] CBT & $P$ & 24 & 30 & 0.94 \\
\hline [125] CBT & $\Sigma$ & 15 & 50 & 1.73 \\
\hline
\end{tabular}

strained by the total cross-sections for $\pi^{-} p \rightarrow n \pi^{+} \pi^{-}$and $\pi^{+} p \rightarrow p \pi^{0} \pi^{0}$ from [126]. Only those $\pi N$ partial waves are included which are required in fits to inelastic channels or which were essential for the discussions in [9] (see table 1). The weights are introduced to guarantee that important data, in particular data on polarization observables, are fitted with good $\chi^{2}$ even at the expense of a slightly worse description of differential cross-sections.

\section{Partial wave analysis and definitions}

The partial wave analysis method used in this analysis is described in detail in $[127,128]$. A shorter survey can be found in [7]. A survey of alternative contemporary partial wave analyses can be found in [7]. In table 7 below we give pole parameters as well as Breit-Wigner parameters. Here, we give the precise definitions used to calculate the quantities given in the tables.

The transition amplitude for a pion- or photoproduced reaction from the initial state $a=\pi N$ or $\gamma N$ and with $b, e . g ., \Lambda K^{+}$, as final state can be defined as

$$
A_{a b}=K_{a c}(I-i \rho K)_{c b}^{-1},
$$

where $K$ is called $K$-matrix and $\rho$ is the phase space. A single resonance is described by the term

$$
K_{a b}=\frac{g_{a} g_{b}}{M^{2}-s}
$$

with $g_{a}, g_{b}$ being coupling constants. In this case, eq. (1) corresponds to the relativistic Breit-Wigner amplitude

$$
A_{a b}=\frac{g_{a} g_{b}}{M^{2}-s-i \sum_{j} g_{j}^{2} \rho_{j}(s)},
$$

where $M=M_{B W}$ is called Breit-Wigner mass. For $\sum_{j} g_{j}^{2} \rho_{j}(s)$ replaced by $M \Gamma$, we obtain the nonrelativistic Breit-Wigner amplitude.

Decays of resonances may be suppressed by the angular-momentum barrier $q^{L}$ where $q$ is the decay momentum and $L$ the orbital angular momentum. The barrier is suppressed by form factors as suggested by Blatt and Weisskopf [129]. The explicit form we use can be found in appendix $\mathrm{C}$ of [127].

The pole position is defined as zero of the amplitude denominator in the complex plane

$$
M^{2}-s-i \sum_{j} g_{j}^{2} \rho_{j}(s)=0,
$$

and the partial width $\Gamma_{a}$ at $s=M^{2}$ (at the BW mass) is defined as

$$
M \Gamma_{a}=g_{a}^{2} \rho_{a}\left(M^{2}\right) .
$$

The helicity-dependent amplitude for photoproduction of the final state $b$ can be written as

$$
a_{b}^{h}(s)=\frac{A_{B W}^{h} g_{b}}{M^{2}-s-i \sum_{j} g_{j}^{2} \rho_{j}(s)},
$$

where $A_{B W}^{h}$ are photoproduction couplings, e.g., helicity couplings in the helicity basis.

In general, the amplitude contains not only one resonance, and there can be important background contributions. Resonances may even be constructed from the iteration of background terms [130-132]. Dynamical coupledchannels models based on effective Lagrangians provide a microscopical description of the background [133, 134].

Here, resonances and background contributions are combined in a $K$-matrix in the form

$$
K_{a b}=\sum_{\alpha} \frac{g_{a}^{\alpha} g_{b}^{\alpha}}{M_{\alpha}^{2}-s}+f_{a b} .
$$

The background terms $f_{a b}$ can be arbitrary functions of $s$ and describe non-resonant transitions from the initial to the final state. In practice, a constant or a parameterization in the form

$$
f_{a b}=\frac{(a+b \sqrt{s})}{\left(s-s_{0}\right)}
$$

was tested. In most partial waves, a constant background term was sufficient to achieve a good fit. The fit to the $(I) J^{P}=(1 / 2) 1 / 2^{-}$wave required the form (8). For the $(I) J^{P}=(3 / 2) 1 / 2^{-}$wave and for the $P$-wave amplitudes, the background form (8) led to a slight improvement, and some fits were done with, others without this term. Both types of solutions were included in the error analysis.

The position of the pole $\left(M_{\text {pole }}-i \frac{1}{2} \Gamma_{\text {pole }}\right)$ can be found by calculation of the zeros of the denominator of a $K$ matrix amplitude in the complex $s$-plane [135]

$$
\operatorname{det}(I-i \rho K) \prod_{\alpha}\left(M_{\alpha}^{2}-s\right)=0 .
$$


We define the residues for the transition amplitude by the contour integral of the amplitude around the pole position in the energy $(\sqrt{s})$ plane to

$$
\begin{aligned}
\operatorname{Res}(a \rightarrow b) & =\int_{o} \frac{\mathrm{d} \sqrt{s}}{2 \pi i} \sqrt{\rho_{a}} A_{a b}(s) \sqrt{\rho_{b}} \\
& =\frac{1}{2 M_{p}} \sqrt{\rho_{a}\left(M_{p}^{2}\right)} g_{a}^{r} g_{b}^{r} \sqrt{\rho_{b}\left(M_{p}^{2}\right)} .
\end{aligned}
$$

Here $M_{p}$ is the position of the pole (complex number) and $g_{a}^{r}$ are pole couplings. The elastic pole residue is defined as

$$
\operatorname{Res}(\pi N \rightarrow N \pi)=\frac{1}{2 M_{p}}\left(g_{N \pi}^{r}\right)^{2} \rho_{N \pi}\left(M_{p}^{2}\right) .
$$

At the pole position one has a full factorization of the amplitude,

$$
\operatorname{Res}^{2}(a \rightarrow b)=\operatorname{Res}(a \rightarrow a) \times \operatorname{Res}(b \rightarrow b) .
$$

The helicity-dependent amplitude for photoproduction of the final state $b$ is calculated in the framework of $P$-vector approach:

$$
a_{b}^{h}=P_{a}^{h}(I-i \rho K)_{j b}^{-1}
$$

where

$$
P_{a}^{h}=\sum_{\alpha} \frac{A_{\alpha}^{h} g_{a}^{\alpha}}{M_{\alpha}^{2}-s}+F_{a} .
$$

and $A_{\alpha}^{h}$ is photo-coupling of the $K$-matrix pole $\alpha$ and $F_{a}$ is a non-resonant transition. In the resonance pole the photo-couplings are defined as

$$
A^{h} g_{b}^{r}=\int_{o} \frac{\mathrm{d} s}{2 \pi i} a_{b}^{h}(s) .
$$

The helicity amplitudes $A^{1 / 2}, A^{3 / 2}$ (photo-couplings in the helicity basis), the coupling elastic residues, and the residues of the transition amplitudes are complex numbers. They become real and coincide with the conventional helicity amplitudes $A^{1 / 2}, A^{3 / 2}$, to half the elastic width $\Gamma_{N \pi} / 2$, and to the channel coupling $\frac{1}{2} \sqrt{\Gamma_{\mathrm{i}} \Gamma_{\mathrm{f}}}$ if a Breit-Wigner amplitude with constant width is used.

The elastic residue, which is proportional to $\left(g_{N \pi}^{r}\right)^{2} \rho_{N \pi}\left(M_{p}^{2}\right)$, defines $g_{N \pi}^{r}$ up to a sign. This may lead to ambiguities if the phase is not properly defined: assume the phase of elastic residue would be $(180 \pm \epsilon)^{\circ}$ in two analyses. Due to eq. (15), the phase of the helicity amplitude depends on this definition. Since the phases of the elastic pole residue of most resonances are negative, we define in the case of elastic residues with a negative real part the phase of $g_{N \pi}^{r}$ clockwise.

In this article we also give some quantities which are related to properties of a relativistic Breit-Wigner amplitude. We define the Breit-Wigner amplitude by

$$
A_{a b}=\frac{f^{2} g_{a}^{r} g_{b}^{r}}{M_{B W}^{2}-s-i f^{2} \sum_{a}\left|g_{a}^{r}\right|^{2} \rho_{a}(s)},
$$

where $M_{B W}$ and scaling factor $f$ are calculated to reproduce exactly the pole position of the resonance. For a true Breit-Wigner amplitude, $f=1$, and the definition in eq. (16) coincides with the one in eq. (3). In the case of a very fast growing phase volume, the Breit-Wigner mass and width can shift from the pole position by a large amount. For example, the Breit-Wigner mass of the Roper resonance is $60-80 \mathrm{MeV}$ higher than the pole position and its Breit-Wigner width exceeds the pole width by about $150 \mathrm{MeV}$. In the $1600-1700 \mathrm{MeV}$ region, the large phase volume leads to a very large Breit-Wigner widths and an appreciable shift in mass from the pole position (see, for example, [29]) if the $\rho N, \Delta \pi$ (with large $L)$, and $D_{15}(1520) \pi$ decay modes are taken into account explicitly. The visible width, e.g., in the $N \pi$ invariant mass spectrum, remains similar to the Breit-Wigner width. Clearly, the large phase volume effects are highly model dependent and possibly, they are artifacts of the formalism. We therefore decided to extract the Breit-Wigner parameters of resonances above the Roper resonance by approximating the phase volumes for the three-body channels in eq. (16) as $\pi N$ phase volume for the respective partial wave. This procedure conserves the branching ratio between three particle and $\pi N$ channels at the resonance position and at the Breit-Wigner mass.

The Breit-Wigner helicity amplitude is defined as

$$
a_{a}^{h}=\frac{A_{B W}^{h} f g_{b}^{r}}{M_{B W}^{2}-s-i f^{2} \sum_{a}\left|g_{a}^{r}\right|^{2} \rho_{a}(s)},
$$

where $A_{B W}^{h}$ is calculated to reproduce the pion photoproduction residues in the pole. In general this quantum is a complex number. However, for majority of resonances its phase deviates only little from 0 or 180 degrees.

\section{Properties of baryon resonances}

On the subsequent pages we present properties of nucleon and $\Delta$ resonances determined in this work. We give pole parameters: pole position (eq. (9)), the complex helicity amplitudes $A^{1 / 2}$ and $A^{3 / 2}$ (eq. (15)), the elastic pole residue (eq. (11)) and residues for hadronic transition amplitudes (eq. (10)).

The tables also give properties of a relativistic BreitWigner amplitude (eq. (16)), its helicity amplitudes (eq. (17)), partial decay widths (eq. (5)), and branching ratios for the decay into channel $a$ by $\Gamma_{a} / \Gamma$.

A large number of resonances is required to achieve a good description of all data sets. In the region above $2.15 \mathrm{GeV}$, further resonances are introduced with spinparity $J^{P}=1 / 2^{ \pm}$and $3 / 2^{ \pm}$. Their isospin, their masses and their widths are ill defined. Likely, more data are required to define their properties. The $\Delta(1930) 5 / 2^{-}$contribution is small and its properties remain ambiguous as well. We decided not to quote any numbers for these resonances even though they improved the fit. These resonances couple to a variety of different decay modes. All decay modes are allowed in the test phase but when a coupling is compatible with zero, it is frozen to zero in the final fits. The optimum set of parameters is determined in fits to the data of tables $1-6$.

The fit minimizes the total log likelihood defined by

$$
-\ln \mathcal{L}_{\text {tot }}=\left(\frac{1}{2} \sum w_{i} \chi_{i}^{2}-\sum w_{i} \ln \mathcal{L}_{i}\right) \frac{\sum N_{i}}{\sum w_{i} N_{i}},
$$


where the summation over binned data contributes to the $\chi^{2}$ while unbinned data contribute to the likelihoods $\mathcal{L}_{i}$. Data with $p \pi^{0} \pi^{0}$ and $p \pi^{0} \eta$ in the final state - except for those taken with polarized photons - are fitted event by event in order to take into account all possible correlations between the variables. For convenience of the reader, we quote differences in fit quality as $\chi^{2}$ difference, with $\Delta \chi^{2}=-2 \Delta \mathcal{L}_{\text {tot }}$. For new data, the weight is increased from $w_{i}=1$ until a visually acceptable fit is reached. Without weights, low-statistics data, e.g., on polarization variables may be reproduced unsatisfactorily without significant deterioration of the total $\mathcal{L}_{\text {tot }}$. The likelihood function is normalized to avoid an artificial increase in statistics by the weighting factors.

Due to the incomplete data base with few double polarization observables only, the solution of the partial wave analysis is not unequivocal. Depending on the number of poles in the different partial waves and depending on start values of the fit, different minima of similar $\chi^{2}$ are reached. However, most parameters are stable, only a few parameters undergo substantial changes. The solutions which have converged to minima of similar depth are stored; from the distribution of the fit results, typically more than ten, the mean value and the error is deduced. Resonances like $N(1700) 3 / 2^{-}$and $\Delta(1700) 3 / 2^{-}$can both decay via $\Delta(1232) \pi$ into the $p \pi^{0} \pi^{0}$ final state. There are no data in our data base which identify the isospin of a $\Delta(1232) \pi$ contribution except the total cross-sections for $\pi^{-} p \rightarrow n \pi^{+} \pi^{-}$and $\pi^{+} p \rightarrow p \pi^{0} \pi^{0}$ from [126]. Hence we give $2 \sigma$ errors for these decay modes.

In some cases, solutions exist with a distinct minimum forming a new class of results, and leading to a new set of parameters. Often, they cluster into two main solutions, called BG2011-01 and BG2011-02. The most significant difference can be found in the $1 / 2\left(3 / 2^{+}\right)$wave where BG2011-02 finds two close-by resonances: $N(1900) 3 / 2^{+}$, present in both types of solutions with slightly different parameters, and $N(1975) 3 / 2^{+}$, present only in BG201102 . Here, we give the properties of $N(1900) 3 / 2^{+}$only. Sizable differences between the BG2011-01 and BG2011-02 solutions are also observed in the $3 / 2^{-}$(in particular for $\left.N(1700) 3 / 2^{-}\right), 5 / 2^{+}$and $7 / 2^{+}$wave. The different solutions are discussed explicitly in [9]. Here, we give errors which cover both solutions. The two solutions give similar properties for $N(1880) 1 / 2^{+}$except for its helicity amplitude. Here, we list both solutions in table 7 .

The $1700 \mathrm{MeV}$ region is complicated due to the presence of two important thresholds, $N(1520) 3 / 2^{-} \pi$ and $\Sigma K . N(1520) 3 / 2^{-} \pi$ in $S$-wave gives $3 / 2^{+}$quantum numbers; indeed, we find a strong $N(1720) 3 / 2^{+} \rightarrow$ $N(1520) 3 / 2^{-} \pi$ coupling. There seems to be a sizable $N(1720) 3 / 2^{+} \rightarrow \Lambda K$ coupling as well; the latter decay requires $L=1 . N(1710) 1 / 2^{+}$may also have a significant $\Lambda K$ coupling. A detailed study is required of the analytic structure of these two resonances in the threshold region. We have not included $\Delta(1750) 1 / 2^{+}$in table 7 below. We find no trace of evidence for this resonance and doubt that it exists. At present, the results on $\Delta(1940) 3 / 2^{-}$from $\gamma p \rightarrow p 2 \pi^{0}$ and $\gamma p \rightarrow p \pi^{0} \eta$ are not consistent. Also this is- sue needs further studies. At present, we give generous errors.

A few "new" resonances are reported. "New" does not mean that resonances with these quantum numbers and similar masses and widths have not been reported before. But, so far, these resonances have not been included in the Review of Particle Properties. These resonances are

$$
\begin{gathered}
N(1880) \frac{1^{+}}{2}, \quad N(1860) \frac{5^{+}}{2}, \quad N(1895) \frac{1^{-}}{2}, \\
N(1875) \frac{3}{2}, \quad N(2150) \frac{3}{2}, \quad \text { and } \quad N(2060) \frac{3^{-}}{2} .
\end{gathered}
$$

Yet, $N(2150) 3 / 2^{-}$could be the $2^{*}$ resonance $N(2080) 3 / 2^{-}$, and $N(2060) 5 / 2^{-}$could be related to $N(2200) 5 / 2^{-}$, with $2^{*}$ as well, of the Particle Data Group.

The $N(1880) 1 / 2^{+}$resonance was first suggested when data on $\gamma p \rightarrow \Sigma^{+} K_{s}^{0}$ from the CBELSA Collaboration [124] were included in the BnGa partial wave analysis. $N(1975) 3 / 2^{+}$emerges from BnGa2011-02 only; it was first reported in [9]. Early evidence for $N(1860) 5 / 2^{+}$has been reported with Breit-Wigner parameters $\left(M_{\mathrm{BW}} ; \Gamma_{\mathrm{BW}}\right)$ equal to $(1882 \pm 10 ; 95 \pm 20)[2,5], 1903 \pm 87 ; 490 \pm 310)$ [137], and $(1817.7 ; 117.6)[5]$. Evidence for $N(1895) 1 / 2^{-}$has been reported by Höhler et al. [136] giving Breit-Wigner parameters of $M_{\mathrm{BW}}=1880 \pm 20, \Gamma_{\mathrm{BW}}=95 \pm 30 \mathrm{MeV}$ for a pole in the $I\left(J^{P}\right)=1 / 2\left(1 / 2^{-}\right)$wave. Manley et al. [137] found a broad state, $M_{\mathrm{BW}}=1928 \pm 59, \Gamma_{\mathrm{BW}}=$ $414 \pm 157 \mathrm{MeV}$. Vrana et al. [138] reported $M_{\mathrm{BW}}=1822 \pm$ $43, \Gamma_{\mathrm{BW}}=246 \pm 185 \mathrm{MeV}$. A third and a forth pole in the $I\left(J^{P}\right)=1 / 2\left(1 / 2^{-}\right)$wave was suggested in [139]. The third pole was given with mass and width of $M_{\text {pole }}=1733 \mathrm{MeV}$; $\Gamma_{\text {pole }}=180 \mathrm{MeV}$, and in [140] with $M_{\text {pole }}=1745 \pm 80$; $\Gamma_{\text {pole }}=220 \pm 95 \mathrm{MeV}$. The latter pole was also seen by Cutkosky et al. [4] at $M_{\text {pole }}=2150 \pm 70, \Gamma_{\text {pole }}=$ $350 \pm 100 \mathrm{MeV}$ and confirmed by Tiator et al. [139].

In the $\frac{1}{2}\left(\frac{3}{2}^{-}\right)$wave, Cutkosky et al. [4] reported two resonances, the lower mass state at $M_{\mathrm{BW}}=1880 \pm$ $100, \Gamma_{\mathrm{BW}}=180 \pm 60 \mathrm{MeV}$, the higher mass pole at $M_{\mathrm{BW}}=2060 \pm 60, \Gamma_{\mathrm{BW}}=300 \pm 10 \mathrm{MeV}$. Saxon et al. [141] and Bell et al. [142] observed a $\frac{1}{2}\left(\frac{3}{2}^{-}\right)$resonance in the reaction $\pi^{-} p \rightarrow \Lambda K^{0}$ at $(1900 ; 240) \mathrm{MeV}$ and $(1920 ; 320) \mathrm{MeV}$, respectively. Based on SAPHIR data on $\gamma p \rightarrow \Lambda K^{+}$[143], Mart and Bennhold claimed evidence for a $\frac{1}{2}\left(\frac{3}{2}^{-}\right)$resonance at $1895 \mathrm{MeV}$ [144] which was confirmed by us on a richer data base in $[145,146]$, with mass and width of $(1875 \pm 25 ; 80 \pm 20) \mathrm{MeV}$, respectively. The high-mass $N_{3 / 2^{-}}$was also seen in $[145$, $146]$ with $\left(2166_{-50}^{+25} ; \Gamma=300 \pm 65\right) \mathrm{MeV}$ and in [147] with $(2100 \pm 20 ; 200 \pm 50) \mathrm{MeV}$.

Table 7. (Next pages) Summary of results of the BnGa partial wave analysis (BnGa2011-02a). The first blocks give quantities related to the pole of the resonance, the second blocks give Breit-Wigner parameters. Masses, width, and residues are given in $\mathrm{MeV}$. The residues of transition amplitudes are divided by $\Gamma_{\text {pole }} / 2$ and are given in $\%$. Small residues may have an error in the phase exceeding $90^{\circ}$. We list those as not defined. 


\begin{tabular}{|c|c|c|c|c|c|c|c|}
\hline$N(1440) \frac{1}{2}^{+}$ & \multicolumn{2}{|c|}{ or $\quad N(1440) P_{11}$} & & $N(1520) \frac{3}{2}^{-}$ & \multicolumn{2}{|c|}{ or $\quad N(1520) D_{13}$} & \\
\hline \multicolumn{4}{|c|}{$N(1440) \frac{1}{2}^{+}$pole parameters $(\mathrm{MeV})$} & \multicolumn{4}{|c|}{$N(1520) \frac{3}{2}^{-}$pole parameters $(\mathrm{MeV})$} \\
\hline$M_{\text {pole }}$ & $1370 \pm 4$ & $\Gamma_{\text {pole }}$ & $190 \pm 7$ & $M_{\text {pole }}$ & $1507 \pm 3$ & $\Gamma_{\mathrm{pole}}$ & $111 \pm 5$ \\
\hline Elastic pole residu & $48 \pm 3$ & Phase & $-(78 \pm 4)^{\circ}$ & Elastic pole residue & $36 \pm 3$ & Phase & $-(14 \pm 3)^{\circ}$ \\
\hline $2 \operatorname{Res}_{\pi N \rightarrow N \sigma} / \Gamma$ & $21 \pm 5 \%$ & Phase & $-(135 \pm 7)^{\circ}$ & $2 \operatorname{Res}_{\pi N \rightarrow \Delta \pi, L=0} / \Gamma$ & $33 \pm 5 \%$ & Phase & $(150 \pm 20)^{\circ}$ \\
\hline $2 \operatorname{Res}_{\pi N \rightarrow \Delta \pi} / \Gamma$ & $27 \pm 2 \%$ & Phase & $(40 \pm 5)^{\circ}$ & $2 \operatorname{Res}_{\pi N \rightarrow \Delta \pi, L=2} / \Gamma$ & $25 \pm 3 \%$ & Phase & $(100 \pm 20)^{\circ}$ \\
\hline \multirow{2}{*}{$A^{1 / 2}\left(\mathrm{GeV}^{-\frac{1}{2}}\right)$} & \multirow{2}{*}{$0.044 \pm 0.007$} & \multirow[t]{2}{*}{ Phase } & \multirow{2}{*}{$(142 \pm 5)^{\circ}$} & \multirow{2}{*}{\multicolumn{3}{|c|}{$\begin{array}{lrl}A^{1 / 2}\left(\mathrm{GeV}^{-\frac{1}{2}}\right) & -0.021 \pm 0.004 & \text { Phase } \\
A^{3 / 2}\left(\mathrm{GeV}^{-\frac{1}{2}}\right) & 0.132 \pm 0.009 & \text { Phase }\end{array}$}} & $(0 \pm 5)^{\circ}$ \\
\hline & & & & & & & $(2 \pm 4)^{\circ}$ \\
\hline \multicolumn{4}{|c|}{$N(1440) \frac{1}{2}^{+}$Breit-Wigner parameters $(\mathrm{MeV})$} & \multicolumn{4}{|c|}{$N(1520) \frac{3}{2}^{-}$Breit-Wigner parameters $(\mathrm{MeV})$} \\
\hline$M_{\mathrm{BW}}$ & $1430 \pm 8$ & $\Gamma_{\mathrm{BW}}$ & $365 \pm 35$ & $M_{\mathrm{BW}}$ & $1517 \pm 3$ & $\Gamma_{\mathrm{BW}}$ & $114 \pm 5$ \\
\hline $\operatorname{Br}(\pi N)$ & $62 \pm 3 \%$ & & & $\operatorname{Br}(\pi N)$ & $62 \pm 3 \%$ & & \\
\hline $\operatorname{Br}(N \sigma)$ & $17 \pm 7 \%$ & $\operatorname{Br}(\Delta \pi)$ & $21 \pm 8 \%$ & $\operatorname{Br}\left(\Delta \pi_{L=0}\right)$ & $19 \pm 4 \%$ & $\operatorname{Br}\left(\Delta \pi_{L=2}\right)$ & $9 \pm 2 \%$ \\
\hline \multicolumn{4}{|c|}{$A_{B W}^{1 / 2}\left(\mathrm{GeV}^{-\frac{1}{2}}\right)-0.061 \pm 0.008$} & \multicolumn{2}{|c|}{$A_{B W}^{1 / 2}\left(\mathrm{GeV}^{-\frac{1}{2}}\right)-0.022 \pm 0.004$} & \multicolumn{2}{|c|}{$A_{B W}^{3 / 2}\left(\mathrm{GeV}^{-\frac{1}{2}}\right) 0.131 \pm 0.010$} \\
\hline$N(1535) \frac{1}{2}^{-}$ & \multicolumn{2}{|c|}{ or $\quad N(1535) S_{11}$} & & $N(1650) \frac{1}{2}^{-}$ & \multicolumn{2}{|c|}{ or $\quad N(1650) S_{11}$} & \\
\hline \multicolumn{4}{|c|}{$N(1535) \frac{1}{2}^{-}$pole parameters $(\mathrm{MeV})$} & \multicolumn{4}{|c|}{$N(1650) \frac{1}{2}^{-}$pole parameters $(\mathrm{MeV})$} \\
\hline$M_{\text {pole }}$ & $1501 \pm 4$ & $\Gamma_{\text {pole }}$ & $134 \pm 11$ & $M_{\text {pole }}$ & $1647 \pm 6$ & $\Gamma_{\text {pole }}$ & $103 \pm 8$ \\
\hline Elastic pole residu & $31 \pm 4$ & Phase & $-(29 \pm 5)^{\circ}$ & Elastic pole residue & $24 \pm 3$ & Phase & $-(75 \pm 12)^{\circ}$ \\
\hline $2 \operatorname{Res}_{\pi N \rightarrow N \eta} / \Gamma$ & $43 \pm 3 \%$ & Phase & $-(76 \pm 5)^{\circ}$ & $2 \operatorname{Res}_{\pi N \rightarrow N \eta} / \Gamma$ & $29 \pm 3 \%$ & Phase & $(134 \pm 10)^{\circ}$ \\
\hline $2 \operatorname{Res}_{\pi N \rightarrow \Delta \pi} / \Gamma$ & $12 \pm 3 \%$ & Phase & $(145 \pm 17)^{\circ}$ & $2 \operatorname{Res}_{\pi N \rightarrow \Lambda K} / \Gamma$ & $23 \pm 9 \%$ & Phase & $(85 \pm 9)^{\circ}$ \\
\hline & & & & $2 \operatorname{Res}_{\pi N \rightarrow \Delta \pi} / \Gamma$ & $23 \pm 4 \%$ & Phase & $-(30 \pm 20)^{\circ}$ \\
\hline$A^{1 / 2}\left(\mathrm{GeV}^{-\frac{1}{2}}\right)$ & $0.116 \pm 0.010$ & Phase & $(7 \pm 6)^{\circ}$ & $A^{1 / 2}\left(\mathrm{GeV}^{-\frac{1}{2}}\right)$ & $33 \pm 0.007$ & Phase & $-(9 \pm 15)^{\circ}$ \\
\hline \multicolumn{4}{|c|}{$N(1535) \frac{1}{2}^{-}$Breit-Wigner parameters $(\mathrm{MeV})$} & \multicolumn{4}{|c|}{$N(1650) \frac{1}{2}^{-}$Breit-Wigner parameters $(\mathrm{MeV})$} \\
\hline$M_{\mathrm{BW}}$ & $1519 \pm 5$ & $\Gamma_{\mathrm{BW}}$ & $128 \pm 14$ & $M_{\mathrm{BW}}$ & $1651 \pm 6$ & $\Gamma_{\mathrm{BW}}$ & $104 \pm 10$ \\
\hline $\operatorname{Br}(\pi N)$ & $54 \pm 5 \%$ & & & $\operatorname{Br}(N \pi)$ & $51 \pm 4 \%$ & $\operatorname{Br}(N \eta)$ & $18 \pm 4 \%$ \\
\hline $\operatorname{Br}(N \eta)$ & $33 \pm 5 \%$ & $\operatorname{Br}(\Delta \pi)$ & $2.5 \pm 1.5 \%$ & $\operatorname{Br}(\Lambda K)$ & $10 \pm 5 \%$ & $\operatorname{Br}(\Delta \pi)$ & $19 \pm 9 \%$ \\
\hline \multicolumn{4}{|c|}{$A_{B W}^{1 / 2}\left(\mathrm{GeV}^{-\frac{1}{2}}\right) \quad 0.105 \pm 0.010$} & \multicolumn{4}{|c|}{$A_{B W}^{1 / 2}\left(\mathrm{GeV}^{-\frac{1}{2}}\right) \quad 0.033 \pm 0.007$} \\
\hline$N(1675) \frac{5}{2}^{-}$ & \multicolumn{3}{|c|}{ or $\quad N(1675) D_{15}$} & $N(1680) \frac{5}{2}^{+}$ & \multicolumn{2}{|c|}{ or $\quad N(1680) F_{15}$} & \\
\hline$N(1675) \frac{5}{2}^{-}$pole $\mathrm{p}$ & parameters $(\mathrm{N}$ & {$[\mathrm{eV})$} & & $N(1680) \frac{5}{2}^{+}$pole par & ameters $(1$ & $\mathrm{eV})$ & \\
\hline$M_{\text {pole }}$ & $1654 \pm 4$ & $\Gamma_{\text {pole }}$ & $151 \pm 5$ & $M_{\text {pole }}$ & $1676 \pm 6$ & $\Gamma_{\text {pole }}$ & $113 \pm 4$ \\
\hline Elastic pole residu & $28 \pm 1$ & Phase & $-(26 \pm 4)^{\circ}$ & Elastic pole residue & $43 \pm 4$ & Phase & $-(2 \pm 10)^{\circ}$ \\
\hline $2 \operatorname{Res}_{\pi N \rightarrow \Delta \pi} / \Gamma$ & $33 \pm 5 \%$ & Phase & $(82 \pm 10)^{\circ}$ & $2 \operatorname{Res}_{\pi N \rightarrow \Delta \pi_{L=1}} / \Gamma$ & $15 \pm 3 \%$ & Phase & $-(70 \pm 45)^{\circ}$ \\
\hline $2 \operatorname{Res}_{\pi N \rightarrow N \sigma} / \Gamma$ & $15 \pm 4 \%$ & Phase & $(132 \pm 18)^{\circ}$ & $2 \operatorname{Res}_{\pi N \rightarrow \Delta \pi_{L=3}} / \Gamma$ & $23 \pm 4 \%$ & Phase & $(85 \pm 15)^{\circ}$ \\
\hline & & & & $2 \operatorname{Res}_{\pi N \rightarrow N \sigma} / \Gamma$ & $26 \pm 4 \%$ & Phase & $-(56 \pm 15)^{\circ}$ \\
\hline$A^{1 / 2}\left(\mathrm{GeV}^{-\frac{1}{2}}\right)$ & $0.024 \pm 0.003$ & Phase & $-(16 \pm 5)^{\circ}$ & $A^{1 / 2}\left(\mathrm{GeV}^{-\frac{1}{2}}\right)-0.0$ & $13 \pm 0.004$ & Phase & $-(25 \pm 22)^{\circ}$ \\
\hline$A^{3 / 2}\left(\mathrm{GeV}^{-\frac{1}{2}}\right)$ & $0.026 \pm 0.008$ & Phase & $-(19 \pm 6)^{\circ}$ & $A^{3 / 2}\left(\mathrm{GeV}^{-\frac{1}{2}}\right) \quad 0.1$ & $34 \pm 0.005$ & Phase & $-(2 \pm 4)^{\circ}$ \\
\hline$N(1675) \frac{5}{2}^{-}$Breit- & -Wigner para & neters $(\mathrm{M}$ & & $N(1680) \frac{5}{2}^{+}$Breit-W & igner para & neters $(\mathrm{MeV}$ & \\
\hline$M_{\mathrm{BW}}$ & $1664 \pm 5$ & $\Gamma_{\mathrm{BW}}$ & $152 \pm 7$ & $M_{\mathrm{BW}}$ & $1689 \pm 6$ & $\Gamma_{\mathrm{BW}}$ & $118 \pm 6$ \\
\hline $\operatorname{Br}(N \pi)$ & $40 \pm 3 \%$ & & & $\operatorname{Br}(N \pi)$ & $64 \pm 5 \%$ & $\operatorname{Br}(N \sigma)$ & $14 \pm 7 \%$ \\
\hline $\operatorname{Br}(\Delta \pi)$ & $33 \pm 8 \%$ & $\operatorname{Br}(N \sigma)$ & $7 \pm 3 \%$ & $\operatorname{Br}\left(\Delta \pi_{L=1}\right)$ & $5 \pm 3 \%$ & $\operatorname{Br}\left(\Delta \pi_{L=3}\right)$ & $10 \pm 3 \%$ \\
\hline$A_{B W}^{1 / 2}\left(\mathrm{GeV}^{-\frac{1}{2}}\right) \quad 0$ & $0.024 \pm 0.003$ & $A_{B W}^{3 / 2}$ & $0.025 \pm 0.007$ & $A_{B W}^{1 / 2}\left(\mathrm{GeV}^{-\frac{1}{2}}\right)-0.0$ & $13 \pm 0.003$ & $A_{B W}^{3 / 2}(\mathrm{GeV}$ & $0.135 \pm 0.006$ \\
\hline
\end{tabular}




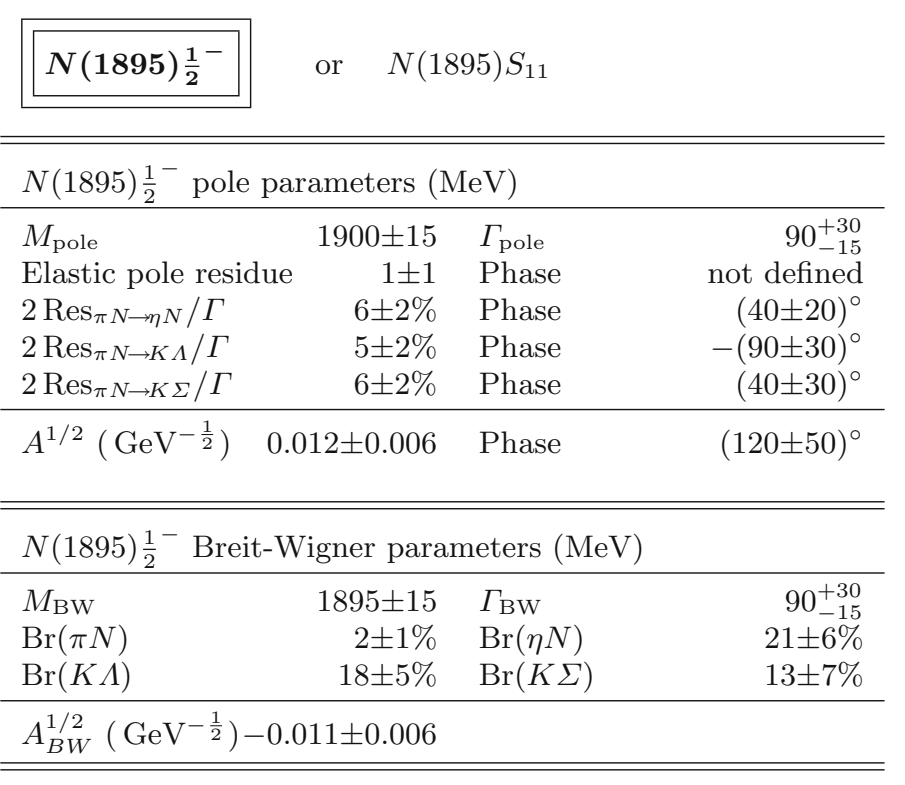

\begin{tabular}{|c|c|c|c|}
\hline$N(1990) \frac{7}{2}^{+}$ & \multicolumn{2}{|c|}{ or $\quad N(1990) F_{17}$} & \\
\hline \multicolumn{4}{|c|}{$N(1990) \frac{7}{2}^{+}$pole parameters $(\mathrm{MeV})$} \\
\hline $\begin{array}{l}M_{\text {pole }} \\
\text { Elastic pole resid }\end{array}$ & $\begin{array}{r}2030 \pm 65 \\
2 \pm 1\end{array}$ & $\begin{array}{l}\Gamma_{\text {pole }} \\
\text { Phase }\end{array}$ & $\begin{array}{r}240 \pm 60 \\
(125 \pm 65)^{\circ}\end{array}$ \\
\hline$A^{1 / 2}\left(\mathrm{GeV}^{-\frac{1}{2}}\right)$ & $0.042 \pm 0.014$ & Phase & $-(30 \pm 20)^{\circ}$ \\
\hline$A^{3 / 2}\left(\mathrm{GeV}^{-\frac{1}{2}}\right)$ & $0.058 \pm 0.012$ & Phase & $-(35 \pm 25)^{\circ}$ \\
\hline \multicolumn{4}{|c|}{$N(1990) \frac{7}{2}^{+}$Breit-Wigner parameters $(\mathrm{MeV})$} \\
\hline $\begin{array}{l}M_{\mathrm{BW}} \\
\operatorname{Br}(\pi N)\end{array}$ & $\begin{array}{r}2060 \pm 65 \\
2 \pm 1 \% \\
\end{array}$ & $\Gamma_{\mathrm{BW}}$ & $240 \pm 50$ \\
\hline$A_{B W}^{1 / 2}\left(\mathrm{GeV}^{-\frac{1}{2}}\right)$ & $0.040 \pm 0.012$ & $A_{B W}^{3 / 2}$ & $0.057 \pm 0.012$ \\
\hline
\end{tabular}

\begin{tabular}{lrlr}
\hline \multicolumn{1}{|c|}{ or $(\mathbf{2 0 6 0}) \frac{\mathbf{5}}{\mathbf{2}}^{-}$} & & & \\
\hline & & \\
\hline
\end{tabular}

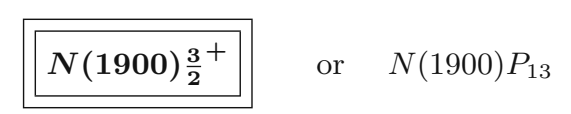

\begin{tabular}{|c|c|c|}
\hline \multicolumn{3}{|c|}{$N(1900) \frac{3}{2}^{+}$pole parameters $(\mathrm{MeV})$} \\
\hline$M_{\text {pole }} \quad 1900 \pm 30$ & $\Gamma_{\text {pole }}$ & $260_{-60}^{+100}$ \\
\hline Elastic pole residue $\quad 3 \pm 2$ & Phase & $(10 \pm 35)^{\circ}$ \\
\hline $2 \operatorname{Res}_{\pi N \rightarrow \eta N} / \Gamma \quad 5 \pm 2 \%$ & Phase & $(70 \pm 60)^{\circ}$ \\
\hline $2 \operatorname{Res}_{\pi N \rightarrow K \Lambda} / \Gamma$ & Phase & $(135 \pm 25)^{\circ}$ \\
\hline $2 \operatorname{Res}_{\pi N \rightarrow K \Sigma} / \Gamma$ & Phase & $(110 \pm 30)^{\circ}$ \\
\hline$A^{1 / 2}\left(\mathrm{GeV}^{-\frac{1}{2}}\right) 0.026 \pm 0.015$ & Phase & $(60 \pm 40)^{\circ}$ \\
\hline$A^{3 / 2}\left(\mathrm{GeV}^{-\frac{1}{2}}\right) 0.060 \pm 0.030$ & Phase & $(185 \pm 60)^{\circ}$ \\
\hline \multicolumn{3}{|c|}{$N(1900) \frac{3}{2}^{+}$Breit-Wigner parameters $(\mathrm{MeV})$} \\
\hline $1905 \pm 30$ & $\Gamma_{\mathrm{BW}}$ & $250_{-50}^{+120}$ \\
\hline $\operatorname{Br}(\pi N)$ & $\operatorname{Br}(\eta N)$ & $10 \pm 4 \%$ \\
\hline $\operatorname{Br}(K \Lambda)$ & $\operatorname{Br}(K \Sigma)$ & $5 \pm 2 \%$ \\
\hline$A_{B W}^{1 / 2}\left(\mathrm{GeV}^{-\frac{1}{2}}\right) 0.026 \pm 0.015$ & $A_{B W}^{3 / 2}(\mathrm{G}$ & $.065 \pm 0.030$ \\
\hline
\end{tabular}

\section{$N(\mathbf{2 0 0 0}) \frac{\mathbf{5}}{\mathbf{2}}^{+} \quad$ or $\quad N(2000) F_{15}$}

\begin{tabular}{|c|c|c|}
\hline \multicolumn{3}{|c|}{$N(2000) \frac{5}{2}^{+}$pole parameters $(\mathrm{MeV})$} \\
\hline $\begin{array}{lr}M_{\text {pole }} & 2030 \pm 110 \\
\text { Elastic pole residue } & 35_{-15}^{+80}\end{array}$ & $\begin{array}{l}\Gamma_{\text {pole }} \\
\text { Phase }\end{array}$ & $\begin{array}{r}480 \pm 100 \\
-(100 \pm 40)^{\circ}\end{array}$ \\
\hline 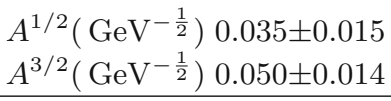 & $\begin{array}{l}\text { Phase } \\
\text { Phase }\end{array}$ & $\begin{array}{r}(15 \pm 40)^{\circ} \\
-(130 \pm 40)^{\circ}\end{array}$ \\
\hline \multicolumn{3}{|c|}{$N(2000) \frac{5}{2}^{+}$Breit-Wigner parameters $(\mathrm{MeV})$} \\
\hline $\begin{array}{r}2090 \pm 120 \\
9 \pm 4 \%\end{array}$ & $\Gamma_{\mathrm{BW}}$ & $460 \pm 100$ \\
\hline$A_{B W}^{1 / 2}\left(\mathrm{GeV}^{-\frac{1}{2}}\right) 0.032 \pm 0.014$ & $A_{B W}^{3 / 2}\left(\mathrm{GeV}^{-\frac{1}{2}}\right)$ & $0.048 \pm 0.014$ \\
\hline
\end{tabular}

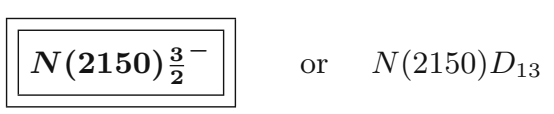

\begin{tabular}{|c|c|c|}
\hline \multicolumn{3}{|c|}{$N(2150) \frac{3}{2}^{-}$pole parameters $(\mathrm{MeV})$} \\
\hline $\begin{array}{lr}M_{\text {pole }} & 2110 \pm 50 \\
\text { Elastic pole residue } & 13 \pm 3 \\
2 \operatorname{Res}_{\pi N \rightarrow K \Lambda / \Gamma} & 3 \pm 1 \% \\
2 \operatorname{Res}_{\pi N \rightarrow K \Sigma / \Gamma} & 2 \pm 1.5 \%\end{array}$ & $\begin{array}{l}\Gamma_{\text {pole }} \\
\text { Phase } \\
\text { Phase } \\
\text { Phase }\end{array}$ & $\begin{array}{r}340 \pm 45 \\
-(20 \pm 10)^{\circ} \\
(100 \pm 30)^{\circ} \\
-(50 \pm 40)^{\circ}\end{array}$ \\
\hline 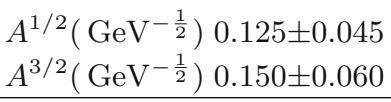 & $\begin{array}{l}\text { Phase } \\
\text { Phase }\end{array}$ & $\begin{array}{l}-(55 \pm 20)^{\circ} \\
-(35 \pm 15)^{\circ}\end{array}$ \\
\hline \multicolumn{3}{|c|}{$N(2150) \frac{3}{2}^{-}$Breit-Wigner parameters $(\mathrm{MeV})$} \\
\hline $\begin{array}{r}2150 \pm 60 \\
6 \pm 2 \%\end{array}$ & $\Gamma_{\mathrm{BW}}$ & $330 \pm 45$ \\
\hline$A_{B W}^{1 / 2}\left(\mathrm{GeV}^{-\frac{1}{2}}\right) 0.130 \pm 0.045$ & $A_{B W}^{3 / 2}\left(\mathrm{GeV}^{-\frac{1}{2}}\right)$ & $0.150 \pm 0.055$ \\
\hline
\end{tabular}




\begin{tabular}{|c|c|c|c|}
\hline$N(2190) \frac{7}{2}^{-}$ & \multicolumn{3}{|c|}{ or $\quad N(2190) G_{17}$} \\
\hline \multicolumn{4}{|c|}{$N(2190) \frac{7}{2}^{-}$pole parameters $(\mathrm{MeV})$} \\
\hline$M_{\text {pole }}$ & $2150 \pm 25$ & $\Gamma_{\text {pole }}$ & $330 \pm 30$ \\
\hline Elastic pole resid & lue $\quad 30 \pm 5$ & Phase & $(30 \pm 10)^{\circ}$ \\
\hline $2 \operatorname{Res}_{\pi N \rightarrow K \Lambda} / \Gamma$ & $3 \pm 1 \%$ & Phase & $(20 \pm 15)^{\circ}$ \\
\hline$A^{1 / 2}\left(\mathrm{GeV}^{-\frac{1}{2}}\right)$ & $0.063 \pm 0.007$ & Phase & $-(170 \pm 15)^{\circ}$ \\
\hline$A^{3 / 2}\left(\mathrm{GeV}^{-\frac{1}{2}}\right)$ & $0.035 \pm 0.020$ & Phase & $(25 \pm 10)^{\circ}$ \\
\hline \multicolumn{4}{|c|}{$N(2190) \frac{7}{2}^{-}$Breit-Wigner parameters $(\mathrm{MeV})$} \\
\hline$M_{\mathrm{BW}}$ & $2180 \pm 20$ & $\Gamma_{\mathrm{BW}}$ & $335 \pm 40$ \\
\hline $\operatorname{Br}(\pi N)$ & $16 \pm 2 \%$ & $\operatorname{Br}(K \Lambda)$ & $0.5 \pm 0.3 \%$ \\
\hline$A_{B W}^{1 / 2}\left(\mathrm{GeV}^{-\frac{1}{2}}\right)-$ & $0.065 \pm 0.008$ & $A_{B W}^{3 / 2}\left(\mathrm{GeV}^{-\frac{1}{2}}\right)$ & $0.035 \pm 0.017$ \\
\hline
\end{tabular}

\begin{tabular}{|c|c|c|c|}
\hline$N(2220) \frac{9}{2}^{+}$ & \multicolumn{2}{|c|}{ or $\quad N(2220) H_{19}$} & \\
\hline \multicolumn{4}{|c|}{$N(2220) \frac{9}{2}^{+}$pole parameters $(\mathrm{MeV})$} \\
\hline $\begin{array}{l}M_{\text {pole }} \\
\text { Elastic pole residue }\end{array}$ & $\begin{array}{r}2150 \pm 35 \\
60 \pm 12\end{array}$ & $\begin{array}{l}\Gamma_{\text {pole }} \\
\text { Phase }\end{array}$ & $\begin{array}{r}440 \pm 40 \\
-(58 \pm 12)^{\circ}\end{array}$ \\
\hline $\begin{array}{l}A^{1 / 2}\left(\mathrm{GeV}^{-\frac{1}{2}}\right) \\
A^{3 / 2}\left(\mathrm{GeV}^{-\frac{1}{2}}\right)\end{array}$ & $\begin{array}{l}<0.010 \\
<0.010\end{array}$ & $\begin{array}{l}\text { Phase } \\
\text { Phase }\end{array}$ & $\begin{array}{l}\text { not defined } \\
\text { not defined }\end{array}$ \\
\hline \multicolumn{4}{|c|}{$N(2220) \frac{9}{2}^{+}$Breit-Wigner parameters $(\mathrm{MeV})$} \\
\hline $\begin{array}{l}M_{\mathrm{BW}} \\
\operatorname{Br}(\pi N)\end{array}$ & $\begin{array}{r}2200 \pm 50 \\
24 \pm 5 \%\end{array}$ & $\Gamma_{\mathrm{BW}}$ & $480 \pm 60$ \\
\hline \multicolumn{2}{|c|}{$\left|A_{B W}^{1 / 2}\right|\left(\mathrm{GeV}^{-\frac{1}{2}}\right)<0.010$} & $\left|A_{B W}^{3 / 2}\right|$ & $<0.010$ \\
\hline
\end{tabular}

\section{$\boldsymbol{N}(\mathbf{2 2 5 0}) \frac{\mathbf{9}}{\mathbf{2}}^{-} \quad$ or $\quad N(2250) G_{19}$}

\begin{tabular}{lrlr}
\hline \hline$N(2250) \frac{9}{2}^{-}$pole parameters $(\mathrm{MeV})$ & \\
\hline$M_{\text {pole }}$ & $2195 \pm 45$ & $\Gamma_{\text {pole }}$ & $470 \pm 50$ \\
Elastic pole residue & $26 \pm 5$ & Phase & $-(38 \pm 25)^{\circ}$ \\
\hline$A^{1 / 2}\left(\mathrm{GeV}^{-\frac{1}{2}}\right)$ & $<0.010$ & Phase & not defined \\
$A^{3 / 2}\left(\mathrm{GeV}^{-\frac{1}{2}}\right)$ & $<0.010$ & Phase & not defined \\
\hline \hline$N(2250) \frac{9}{2}^{-}$Breit-Wigner parameters $(\mathrm{MeV})$ \\
\hline$M_{\mathrm{BW}} 2280 \pm 40$ & $\Gamma_{\mathrm{BW}}$ \\
$\operatorname{Br}(\pi N)$ & $12 \pm 4 \%$ \\
\hline$\left|A_{B W}^{1 / 2}\right|\left(\mathrm{GeV}^{-\frac{1}{2}}\right)<0.010$ & $\left|A_{B W}^{3 / 2}\right|\left(\mathrm{GeV}^{-\frac{1}{2}}\right)<0.010$ \\
\hline \hline
\end{tabular}

$\Delta(\mathbf{1 2 3 2}) \frac{\mathbf{3}}{\mathbf{2}}^{+} \quad$ or $\quad \Delta(1232) P_{33}$

\begin{tabular}{|c|c|c|}
\hline \multicolumn{3}{|c|}{$\Delta(1232) \frac{3}{2}^{+}$pole parameters $(\mathrm{MeV})$} \\
\hline $\begin{array}{ll}M_{\text {pole }} & 1210.5 \pm 1.0\end{array}$ & $\Gamma_{\text {pole }}$ & $99 \pm 2$ \\
\hline Elastic pole residue $51.6 \pm 0.6$ & Phase & $-(46 \pm 1)^{\circ}$ \\
\hline
\end{tabular}

\begin{tabular}{llr}
\hline$A^{1 / 2}\left(\mathrm{GeV}^{-\frac{1}{2}}\right)-0.131 \pm 0.0035$ & Phase & $-(19 \pm 2)^{\circ}$ \\
$A^{3 / 2}\left(\mathrm{GeV}^{-\frac{1}{2}}\right)-0.254 \pm 0.0045$ & Phase & $-(9 \pm 1)^{\circ}$ \\
\hline \hline
\end{tabular}

$\Delta(1232) \frac{3}{2}^{-}$Breit-Wigner parameters $(\mathrm{MeV})$

\begin{tabular}{lll}
\hline$M_{\mathrm{BW}}$ & $1228 \pm 2$ & $\Gamma_{\mathrm{BW}}$ \\
& & $110 \pm 3$ \\
& & \\
\hline$A_{B W}^{1 / 2}\left(\mathrm{GeV}^{-\frac{1}{2}}\right)-0.131 \pm 0.004$ & $A_{B W}^{3 / 2}\left(\mathrm{GeV}^{-\frac{1}{2}}\right)-0.254 \pm 0.005$ \\
\hline \hline
\end{tabular}

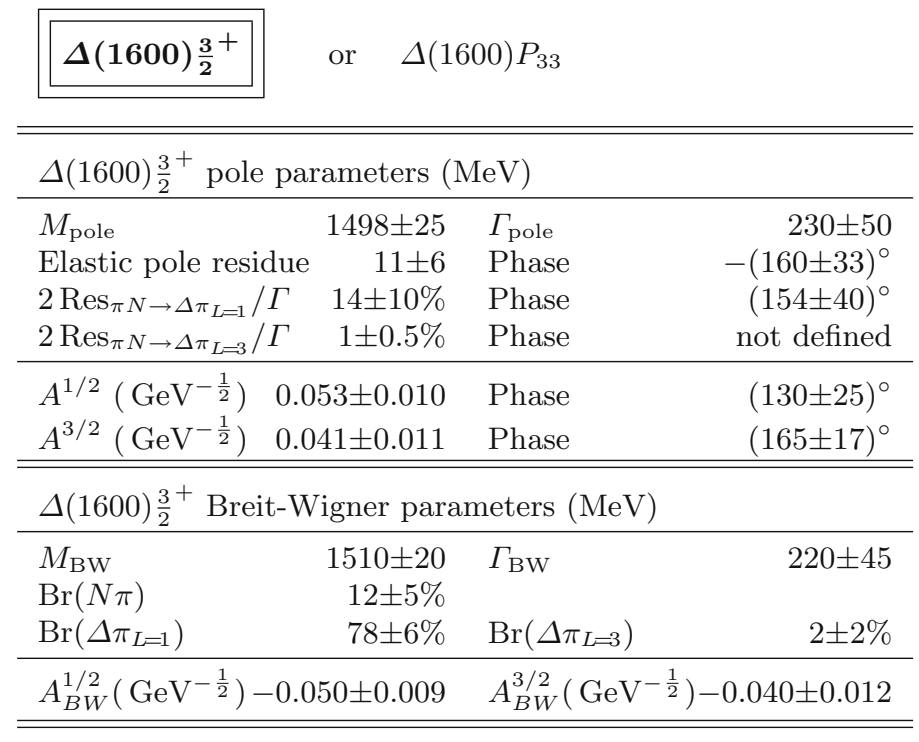




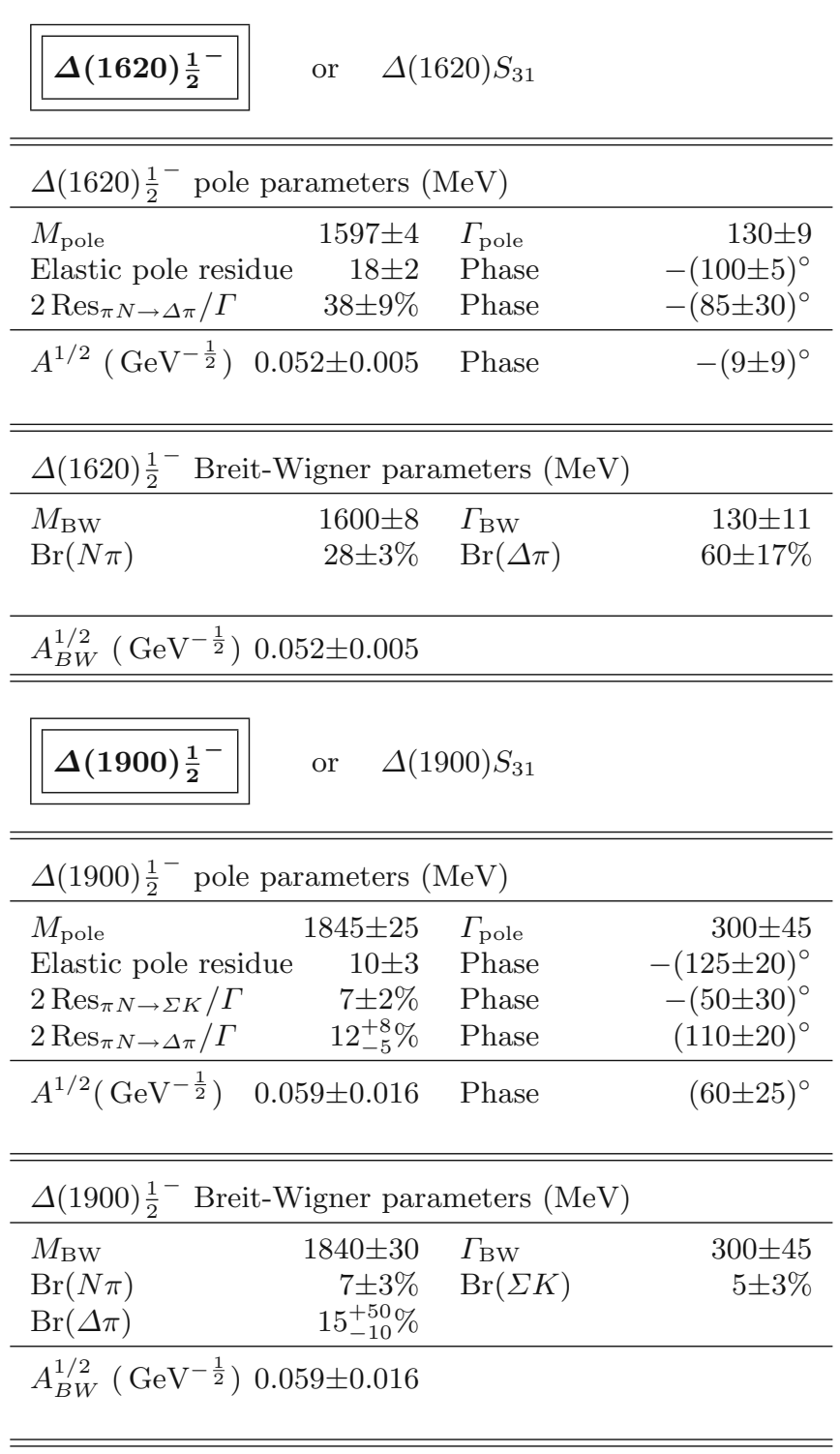

\begin{tabular}{|c|c|c|c|}
\hline$\Delta(1910) \frac{1}{2}^{+}$ & \multicolumn{2}{|c|}{ or $\quad \Delta(1910) P_{31}$} & \\
\hline \multicolumn{4}{|c|}{$\Delta(1910) \frac{1}{2}^{+}$pole parameters $(\mathrm{MeV})$} \\
\hline $\begin{array}{l}M_{\text {pole }} \\
\text { Elastic pole resid } \\
2 \operatorname{Res}_{\pi N \rightarrow \Sigma K} / \Gamma \\
2 \operatorname{Res}_{\pi N \rightarrow \Delta \pi} / \Gamma\end{array}$ & $\begin{array}{r}1850 \pm 40 \\
24 \pm 6 \\
7 \pm 2 \% \\
16 \pm 9 \% \\
\end{array}$ & $\begin{array}{l}\Gamma_{\text {pole }} \\
\text { Phase } \\
\text { Phase } \\
\text { Phase }\end{array}$ & $\begin{array}{r}350 \pm 45 \\
-(145 \pm 30)^{\circ} \\
-(110 \pm 30)^{\circ} \\
(95 \pm 40)^{\circ} \\
\end{array}$ \\
\hline$A^{1 / 2}\left(\mathrm{GeV}^{-\frac{1}{2}}\right)$ & $0.023 \pm 0.009$ & Phase & $(40 \pm 90)^{\circ}$ \\
\hline \multicolumn{4}{|c|}{$\Delta(1910) \frac{1}{2}^{-}$Breit-Wigner parameters $(\mathrm{MeV})$} \\
\hline $\begin{array}{l}M_{\mathrm{BW}} \\
\operatorname{Br}(N \pi) \\
\operatorname{Br}(\Delta \pi)\end{array}$ & $\begin{array}{c}1860 \pm 40 \\
12 \pm 3 \% \\
60 \pm 28 \%\end{array}$ & $\begin{array}{l}\Gamma_{\mathrm{BW}} \\
\operatorname{Br}(\Sigma K)\end{array}$ & $\begin{array}{r}350 \pm 55 \\
9 \pm 5 \%\end{array}$ \\
\hline$A_{B W}^{1 / 2}\left(\mathrm{GeV}^{-\frac{1}{2}}\right)$ & $0.022 \pm 0.009$ & & \\
\hline
\end{tabular}

\section{$\Delta(\mathbf{1 7 0 0}) \frac{\mathbf{3}}{\mathbf{2}}^{-} \quad$ or $\quad \Delta(1700) D_{33}$}

\begin{tabular}{|c|c|c|c|}
\hline \multicolumn{4}{|c|}{$\Delta(1700) \frac{3}{2}^{-}$pole parameters $(\mathrm{MeV})$} \\
\hline$M_{\text {pole }}$ & $1680 \pm 10$ & $\Gamma_{\text {pole }}$ & $305 \pm 15$ \\
\hline Elastic pole residue & $42 \pm 7$ & Phase & $-(3 \pm 15)^{\circ}$ \\
\hline $2 \operatorname{Res}_{\pi N \rightarrow \Delta \eta} / \Gamma$ & $12 \pm 3 \%$ & Phase & $-(60 \pm 15)^{\circ}$ \\
\hline$A^{1 / 2}\left(\mathrm{GeV}^{-\frac{1}{2}}\right)$ & $0.170 \pm 0.020$ & Phase & $(50 \pm 15)^{\circ}$ \\
\hline$A^{3 / 2}\left(\mathrm{GeV}^{-\frac{1}{2}}\right)$ & $0.170 \pm 0.025$ & Phase & $(45 \pm 10)^{\circ}$ \\
\hline \multicolumn{4}{|c|}{$\Delta(1700) \frac{3}{2}^{-}$Breit-Wigner parameters $(\mathrm{MeV})$} \\
\hline$M_{\mathrm{BW}}$ & $1715_{-15}^{+30}$ & $\Gamma_{\mathrm{BW}}$ & $310_{-15}^{+40}$ \\
\hline $\operatorname{Br}(N \pi)$ & $22 \pm 4 \%$ & $\operatorname{Br}(\Delta \eta)$ & $5 \pm 2 \%$ \\
\hline $\operatorname{Br}\left(\Delta \pi_{L=0}\right)$ & $20_{-13}^{+25} \%$ & $\operatorname{Br}\left(\Delta \pi_{L=2}\right)$ & $12_{-7}^{+14} \%$ \\
\hline$A_{B W}^{1 / 2}\left(\mathrm{GeV}^{-\frac{1}{2}}\right)$ & $0.160 \pm 0.020$ & $A_{B W}^{3 / 2}\left(\mathrm{GeV}^{-\frac{1}{2}}\right)$ & $0.165 \pm 0.025$ \\
\hline
\end{tabular}

\section{$\Delta(\mathbf{1 9 0 5}) \frac{\mathbf{5}}{\mathbf{2}}^{+} \quad$ or $\quad \Delta(1905) F_{35}$}

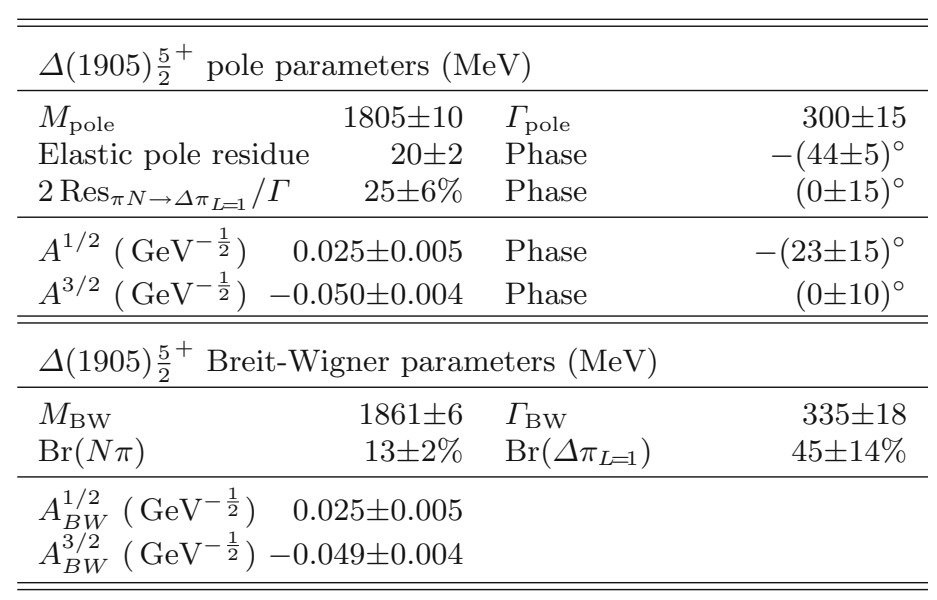

$$
\Delta \text { (1920) } \frac{\mathbf{3}}{\mathbf{2}}^{+} \quad \text { or } \quad \Delta(1920) P_{33}
$$

\begin{tabular}{|c|c|c|c|}
\hline \multicolumn{4}{|c|}{$\Delta(1920) \frac{3}{2}^{+}$pole parameters $(\mathrm{MeV})$} \\
\hline$M_{\text {pole }}$ & $1890 \pm 30$ & $\Gamma_{\text {pole }}$ & $300 \pm 60$ \\
\hline Elastic pole residue & $17 \pm 8$ & Phase & $-(40 \pm 20)^{\circ}$ \\
\hline $2 \operatorname{Res}_{\pi N \rightarrow \Sigma K} / \Gamma$ & $9 \pm 3 \%$ & Phase & $(80 \pm 40)^{\circ}$ \\
\hline $2 \operatorname{Res}_{\pi N \rightarrow \Delta \eta} / \Gamma$ & $17 \pm 8 \%$ & Phase & $(70 \pm 20)^{\circ}$ \\
\hline $2 \operatorname{Res}_{\pi N \rightarrow \Delta \pi_{L=1}} / \Gamma$ & $20 \pm 12 \%$ & Phase & $-(120 \pm 30)^{\circ}$ \\
\hline $2 \operatorname{Res}_{\pi N \rightarrow \Delta \pi_{L=3}} / \Gamma$ & $28 \pm 7 \%$ & Phase & $-(95 \pm 35)^{\circ}$ \\
\hline$A^{1 / 2}\left(\mathrm{GeV}^{-\frac{1}{2}}\right)$ & $0.130_{-0.060}^{+0.030}$ & Phase & $-(65 \pm 20)^{\circ}$ \\
\hline$A^{3 / 2}\left(\mathrm{GeV}^{-\frac{1}{2}}\right)$ & $0.115_{-0.050}^{+0.025}$ & Phase & $-(160 \pm 20)^{\circ}$ \\
\hline \multicolumn{4}{|c|}{$\Delta(1920) \frac{3}{2}^{+}$Breit-Wigner parameters $(\mathrm{MeV})$} \\
\hline$M_{\mathrm{BW}}$ & $1900 \pm 30$ & $\Gamma_{\mathrm{BW}}$ & $310 \pm 60$ \\
\hline $\operatorname{Br}(N \pi)$ & $8 \pm 4 \%$ & $\operatorname{Br}(\Sigma K)$ & $4 \pm 2 \%$ \\
\hline $\operatorname{Br}(\Delta \eta)$ & $15 \pm 8 \%$ & & \\
\hline $\operatorname{Br}\left(\Delta \pi_{L=1}\right)$ & $22 \pm 12 \%$ & $\operatorname{Br}\left(\Delta \pi_{L=3}\right)$ & $45 \pm 20 \%$ \\
\hline$A_{B W}^{1 / 2}\left(\mathrm{GeV}^{-\frac{1}{2}}\right)$ & $0.130_{-0.060}^{+0.030}$ & & \\
\hline$A_{B W}^{3 / 2}\left(\mathrm{GeV}^{-\frac{1}{2}}\right)$ & $-0.115_{-0.050}^{+0.025}$ & & \\
\hline
\end{tabular}




\begin{tabular}{|c|c|c|}
\hline$\Delta(1940) \frac{3}{2}^{-}$ & or $\quad \Delta(1940) D_{33}$ & \\
\hline$\Delta(1940) \frac{3}{2}^{-} \mathrm{pol}$ & parameters $(\mathrm{MeV})$ & \\
\hline $\begin{array}{l}M_{\text {pole }} \\
\text { Elastic pole res }\end{array}$ & 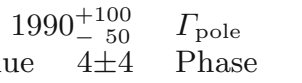 & $450 \pm 90$ \\
\hline
\end{tabular}

\begin{tabular}{lcll}
\hline \hline$\Delta(1940) \frac{3}{2}^{-}$ & Breit-Wigner parameters $(\mathrm{MeV})$ & \\
\hline$M_{\mathrm{BW}}$ & $1995_{-60}^{+105}$ & $\Gamma_{\mathrm{BW}}$ & $450 \pm 100$
\end{tabular}

\section{Significance and rating}

The fits presented here are based on a large number of resonances. Hence one questions arises naturally: can the results be trusted? We are convinced that the answer is yes, because of the predictive power of our amplitudes. After fitting the CLAS $C_{x}, C_{z}$ data [120] we provided predictions for $O_{x}, O_{z}$ from GRAAL [119]. Our main solution agreed with the data as if the data were fitted.

In table 8 we give our rating of the evidence with which baryon resonances are observed. By definition:

$$
\begin{array}{ll}
* * * * \quad \text { Existence is certain, and properties are at least fairly } \\
\text { well explored. } \\
\text { Ex** } \\
\text { ther confirmation is desirable and/or quantum num- } \\
\text { bers, branching fractions etc. are not well determined. } \\
* * \quad \text { Evidence of existence is only fair. } \\
* \quad \text { Evidence of existence is poor. }
\end{array}
$$

The significance of a resonance and of its decay modes is estimated from three sources: i) from the increase in $\chi^{2}$ when a resonance is removed from the fit, both the overall increase in $\chi^{2}$, and the increase in $\chi^{2}$ in specific final states, ii) from the stability of the fit result when the hypothesis (e.g., number of poles in a given partial wave) is changed, and iii) from the errors in the definition of masses, widths, residues, photo-couplings, etc. As a rule we give $1^{*}$ when a decay mode is seen with a significance of at least $2 \sigma, 2^{*}$ for a significance of at least $3.5 \sigma$, and $3^{*}$ for a significance of at least $5 \sigma$. As there are ambiguous solutions, we do not assign $4 *$ for decays derived from photoproduction. In some cases, the errors are large, and the significance is high. This happens, if there are two solutions which give different values for an observable, e.g., for its photoproduction amplitude. Without the resonance, the photoproduction data cannot be described; hence we are sure that the resonance is needed. But the actual value may be less certain. The star rating reflects our estimate on how safe we are in claiming the existence of the resonance from photoproduction data; the error gives the range of values of resonance properties which might

\begin{tabular}{|c|c|c|c|c|c|c|c|}
\hline All & $\pi N$ & $\gamma N$ & $N \eta$ & $\Lambda K$ & $\Sigma K$ & $\Delta \pi$ & $N \sigma$ \\
\hline$N(1440) \frac{1}{2}^{+} * * * *$ & $* * * *$ & $* * * \star$ & $(*)$ & & & $* * *$ & $\star \star \star$ \\
\hline$N(1710) \frac{1}{2}^{+} * * *$ & $* * *$ & $* * *$ & $* * \star$ & $\star \star \star$ & $\star \star$ & $*(*)$ & \\
\hline$N(1880) \frac{1}{2}^{+} \star \star \star$ & $\star$ & $\star$ & & $\star \star$ & $\star$ & & \\
\hline$N(1535) \frac{1}{2}^{-} * * * *$ & $* * * *$ & $* * * *$ & $* * * *$ & & & $*$ & \\
\hline$N(1650) \frac{1}{2}-* * * *$ & $* * * *$ & $* * *$ & $* \star \star$ & $* * *$ & $* *$ & $* *(*)$ & \\
\hline$N(1895) \frac{1}{2}^{-} \quad \star \star$ & $\star$ & $\star \star$ & $\star \star$ & $\star \star$ & $\star$ & & \\
\hline$N(1720) \frac{3}{2}+* * * *$ & $* * * *$ & $* * * *$ & $* * * *$ & $* *$ & $* *$ & $* * *$ & \\
\hline$N(1900) \frac{3}{2}^{+} * * \star$ & $* *$ & $\star \star \star$ & $\star \star$ & $\star \star \star$ & $\star \star$ & $\star \star$ & \\
\hline$N(1520) \frac{3}{2}^{-} * * * *$ & $* * * *$ & $* * * *$ & $* * *$ & & & $* * * *$ & \\
\hline$N(1700) \frac{3}{2}-* * \star$ & $* *$ & $* *$ & $*$ & $*(*)$ & $*$ & $* * \star$ & \\
\hline$N(1875) \frac{3}{2}^{-} \star \star \star$ & $\star$ & $\star \star \star$ & & $\star \star \star$ & $\star \star$ & & $\star \star \star$ \\
\hline$N(2150) \frac{3}{2}^{-} \quad \star \star$ & $\star \star$ & $\star \star$ & & $\star \star$ & & $\star \star$ & \\
\hline$N(1680) \frac{5}{2}^{+} * * * *$ & $* * * *$ & $* * * *$ & $*$ & & & $* *(*)$ & $\star \star$ \\
\hline$N(1860) \frac{5}{2}^{+} \star \star$ & $\star$ & $\star$ & & & & & \\
\hline 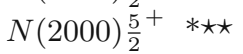 & $*(*)$ & $\star \star$ & $\star \star$ & $\star \star$ & $\star$ & & \\
\hline$N(1675) \frac{5}{2}-* * * *$ & $* * * *$ & $* * *(*)$ & $*$ & $*$ & & $* * *(*)$ & 夫 \\
\hline$N(2060) \frac{5}{2}-\star \star \star$ & $\star \star$ & $\star \star \star$ & $\star$ & & $\star \star$ & & \\
\hline$N(1990) \frac{7}{2}^{+} \quad * *$ & $*(*)$ & $\star \star$ & & & & & \\
\hline$N(2190) \frac{7}{2}^{-} * * * *$ & $* * * *$ & $* \star \star$ & & $\star \star$ & & & \\
\hline$N(2220) \frac{9}{2}^{+} * * * *$ & $* * * *$ & & & & & & \\
\hline$N(2250) \frac{9}{2}^{-} * * * *$ & $* * * *$ & & & & & & \\
\hline$\Delta(1910) \frac{1}{2}^{+} * * * *$ & $* * * *$ & $* \star$ & & & $* \star$ & $* \star$ & \\
\hline$\Delta(1620) \frac{1}{2}^{-} * * * *$ & $* * * *$ & $* * *$ & & & & $* * * *$ & \\
\hline$\Delta(1900) \frac{1}{2}^{-} \quad * *$ & $* *$ & $* \star$ & & & $* \star$ & $* \star$ & \\
\hline$\Delta(1232) \frac{3}{2}^{+} * * * *$ & $* * * *$ & $* * * *$ & & & & & \\
\hline$\Delta(1600) \frac{3}{2}^{+} * * *$ & $* * *$ & $* * \star$ & & & & $* * *$ & \\
\hline$\Delta(1920) \frac{3}{2}^{+} * * *$ & $* * *$ & $* \star$ & & & $* \star \star$ & $* *$ & \\
\hline$\Delta(1700) \frac{3}{2}^{-} * * *$ & $* * *$ & $* * *$ & & & & $* *$ & \\
\hline$\Delta(1940) \frac{3}{2}-\quad *$ & $*$ & $\star \star$ & & & & $\star \star$ fro & $\mathrm{m} \Delta \eta$ \\
\hline$\Delta(1905) \frac{5}{2}{ }^{+} * * * *$ & $* * * *$ & $* * * *$ & & & $* \star \star$ & $* *(* *)$ & \\
\hline$\Delta(1950) \frac{7}{2}^{+} * * * *$ & $* * * *$ & $* * *$ & & & $* \star \star$ & $* * \star$ & \\
\hline
\end{tabular}
be assigned to a given resonance.

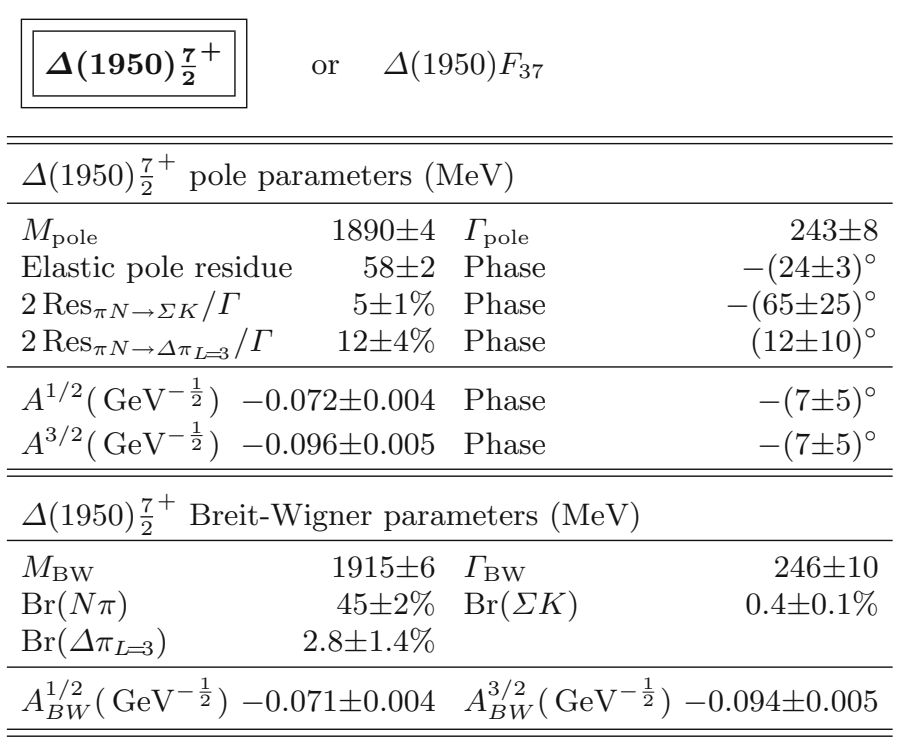

Table 8. Star rating suggested for baryon resonances and their decays. Ratings of the Particle Data Group are given as *; additional stars suggested from this analysis are represented by $\star ;(*)$ stands for stars which should be removed. 
We would like to thank the members of SFB/TR16 for continuous encouragement. We acknowledge support from the Deutsche Forschungsgemeinschaft (DFG) within the SFB/ TR16 and from the Forschungszentrum Jülich within the FFE program.

\section{References}

1. K. Nakamura, J. Phys. G 37, 075021 (2010).

2. G. Höhler, F. Kaiser, R. Koch, E. Pietarinen, Handbook Of Pion Nucleon Scattering (Karlsruhe, 1979) pp. 440 (Physics Data, No. 12-1 (1979)).

3. G. Höhler, $\pi N$ Newslett. 9, 108 (1993).

4. R.E. Cutkosky et al., Pion - Nucleon Partial Wave Analysis, in Proceedings of the 4 th International Conference on Baryon Resonances, Toronto, Canada, Jul 14-16, 1980 (Baryon, 1980) QCD161:C45:1980.

5. R.A. Arndt, W.J. Briscoe, I.I. Strakovsky, R.L. Workman, Phys. Rev. C 74, 045205 (2006).

6. A.V. Anisovich, E. Klempt, V.A. Nikonov, M.A. Matveev, A.V. Sarantsev, U. Thoma, Eur. Phys. J. A 44, 203 (2010).

7. A.V. Anisovich, E. Klempt, V.A. Nikonov, A.V. Sarantsev, U. Thoma, Eur. Phys. J. A 47, 27 (2011).

8. A.V. Anisovich, E. Klempt, V. Kuznetsov, V.A. Nikonov, M.V. Polyakov, A.V. Sarantsev, U. Thoma, arXiv:1108.3010 [hep-ph].

9. A.V. Anisovich, E. Klempt, V.A. Nikonov, A.V. Sarantsev, U. Thoma, Eur. Phys. J. A 47, 153 (2011).

10. M. Fuchs et al., Photoproduction of two $\pi^{0}$ off protons for $0.3<E_{\gamma}<3 \mathrm{GeV}$, in preparation.

11. V. Sokhoyan, arXiv:1108.5283 [nucl-ex].

12. E. Gutz et al., Phys. Lett. B 687, 11 (2010).

13. I. Horn et al., Phys. Rev. Lett. 101, 202002 (2008).

14. W.B. Richards et al., Phys. Rev. D 1, 10 (1970).

15. S. Prakhov et al., Phys. Rev. C 72, 015203 (2005).

16. T.M. Knasel et al., Phys. Rev. D 11, 1 (1975).

17. R.D. Baker et al., Nucl. Phys. B 141, 29 (1978).

18. D.H. Saxon et al., Nucl. Phys. B 162, 522 (1980).

19. K.W. Bell et al., Nucl. Phys. B 222, 389 (1983).

20. D.J. Candlin et al., Nucl. Phys. B 226, 1 (1983).

21. F.S. Crawford, F. Grard, G.A. Smith, Phys. Rev. 128, 368 (1962).

22. M. Winik, S. Toaff, D. Revel, J. Goldberg, L. Berny, Nucl. Phys. B 128, 66 (1977).

23. C. Baltay et al., Rev. Mod. Phys. 33, 374 (1961).

24. N.L. Carayannopoulos et al., Phys. Rev. 138, 433 (1965).

25. E.H. Bellamy et al., Phys. Lett. B 39, 299 (1972).

26. D.J. Candlin et al., Nucl. Phys. B 311, 613 (1989).

27. J.C. Hart et al., Nucl. Phys. B 166, 73 (1980).

28. S. Prakhov et al., Phys. Rev. C 69, 045202 (2004).

29. U. Thoma et al., Phys. Lett. B 659, 87 (2008).

30. A.V. Sarantsev et al., Phys. Lett. B 659, 94 (2008).

31. C. Weinheimer, Nucl. Phys. A 721, 781 (2003).

32. I. Horn et al., Eur. Phys. J. A 38, 173 (2008).

33. Y. Assafiri et al., Phys. Rev. Lett. 90, 222001 (2003).

34. E. Gutz et al., Eur. Phys. J. A 35, 291 (2008).

35. J. Ahrens et al., Eur. Phys. J. A 34, 11 (2007).

36. M. Fuchs et al., Phys. Lett. B 368, 20 (1996).

37. J. Ahrens et al., Phys. Rev. Lett. 88, 232002 (2002).

38. J. Ahrens et al., Eur. Phys. J. A 21, 323 (2004).

39. O. Bartalini et al., Eur. Phys. J. A 26, 399 (2005).
40. O. Bartholomy et al., Phys. Rev. Lett. 94, 012003 (2005).

41. H. van Pee et al., Eur. Phys. J. A 31, 61 (2007).

42. M. Dugger et al., Phys. Rev. C 76, 025211 (2007).

43. V. Crede et al., Phys. Rev. C 84, 055203 (2011).

44. G. Barbiellini et al., Phys. Rev. 184, 1402 (1969).

45. V.G. Gorbenko et al., Pisma Zh. Eksp. Teor. Fiz. 19, 659 (1974).

46. V.G. Gorbenko et al., Yad. Fiz. 27, 1204 (1978).

47. A.A. Belyaev et al., Nucl. Phys. B 213, 201 (1983).

48. G. Blanpied et al., Phys. Rev. Lett. 69, 1880 (1992).

49. R. Beck et al., Phys. Rev. Lett. 78, 606 (1997).

50. F.V. Adamian et al., Phys. Rev. C 63, 054606 (2001).

51. G. Blanpied et al., Phys. Rev. C 64, 025203 (2001).

52. N. Sparks et al., Phys. Rev. C 81, 065210 (2010).

53. P.S.L. Booth et al., Nucl. Phys. B 121, 45 (1977).

54. P. Feller et al., Nucl. Phys. B 110, 397 (1976).

55. V.G. Gorbenko et al., Yad. Fiz. 26, 320 (1977).

56. H. Herr et al., Nucl. Phys. B 125, 157 (1977).

57. M. Fukushima et al., Nucl. Phys. B 136, 189 (1978).

58. P.J. Bussey et al., Nucl. Phys. B 154, 492 (1979).

59. K.S. Agababian et al., Sov. J. Nucl. Phys. 50, 834 (1989) Yad. Fiz. 50, 1341 (1989).

60. M.M. Asaturian et al., JETP Lett. 44, 341 (1986) Pisma Zh. Eksp. Teor. Fiz. 44, 266 (1986).

61. A. Bock et al., Phys. Rev. Lett. 81, 534 (1998).

62. J.O. Maloy, Ph.D. Thesis (1961).

63. V.G. Gorbenko et al., Pisma Zh. Eksp. Teor. Fiz. 22, 393 (1975).

64. S. Kato et al., Nucl. Phys. B 168, 1 (1980).

65. A.S. Bratashevsky et al., Nucl. Phys. B 166, 525 (1980).

66. A.S. Bratashevsky et al., Ukr. Fiz. Zh. 31, 1306 (1986) (Russian edition).

67. P.J. Bussey et al., Nucl. Phys. B 159, 383 (1979).

68. J. Ahrens et al., Eur. Phys. J. A 26, 135 (2005).

69. R.O. Avakyan et al., Sov. J. Nucl. Phys. 53, 448 (1991) Yad. Fiz. 53, 717 (1991).

70. S.D. Ecklund, R.L. Walker, Phys. Rev. 159, 1195 (1967).

71. C. Betourne, J.C. Bizot, J.P. Perez-y-Jorba, D. Treille, W. Schmidt, Phys. Rev. 172, 1343 (1968).

72. B. Bouquet et al., Phys. Rev. Lett. 27, 1244 (1971).

73. T. Fujii et al., Phys. Rev. Lett. 26, 1672 (1971).

74. K. Ekstrand et al., Phys. Rev. D 6, 1 (1972).

75. T. Fujii et al., Nucl. Phys. B 120, 395 (1977).

76. I. Arai et al., J. Phys. Soc. Jpn. 43, 363 (1977).

77. E.J. Durwen, Ph.D. Thesis (1980), BONN-IR-80-7, April 1980.

78. K.H. Althoff et al., Z. Phys. C 18, 199 (1983).

79. W. Heise, Ph.D. Thesis (1988), BONN-IR-88-06, February 1988.

80. K. Buechler et al., Nucl. Phys. A 570, 580 (1994).

81. H.W. Dannhausen et al., Eur. Phys. J. A 11, 441 (2001).

82. J. Ahrens et al., Phys. Rev. C 74, 045204 (2006).

83. M. Dugger et al., Phys. Rev. C 79, 065206 (2009).

84. R.E. Taylor, R.F. Mozley, Phys. Rev. 117, 835 (1960).

85. R.C. Smith, R.F. Mozley, Phys. Rev. 130, 2429 (1963).

86. J. Alspector et al., Phys. Rev. Lett. 28, 1403 (1972).

87. G. Knies et al., Phys. Rev. D 10, 2778 (1974).

88. V.B. Ganenko et al., Yad. Fiz. 23, 100 (1976).

89. P.J. Bussey et al., Nucl. Phys. B 154, 205 (1979).

90. V. A. Getman et al., Nucl. Phys. B 188, 397 (1981).

91. P. Hampe, Ph.D. Thesis, 1980.

92. R. Beck et al., Phys. Rev. C 61, 035204 (2000).

93. J. Ajaka et al., Phys. Lett. B 475, 372 (2000). 
94. J. Bocquet et al., AIP Conf. Proc. 603, 499 (2001).

95. K. H. Althoff et al., Nucl. Phys. B 53, 9 (1973).

96. S. Arai et al., Nucl. Phys. B 48, 397 (1972).

97. P. Feller et al., Phys. Lett. B 52, 105 (1974) Nucl. Phys. B 102, 207 (1976).

98. K.H. Althoff et al., Phys. Lett. B 59, 93 (1975).

99. H. Genzel et al., Nucl. Phys. B 92, 196 (1975).

100. K.H. Althoff et al., Phys. Lett. B 63, 107 (1976).

101. K.H. Althoff et al., Nucl. Phys. B 131, 1 (1977).

102. M. Fukushima et al., Nucl. Phys. B 130, 486 (1977).

103. V.A. Getman et al., Yad. Fiz. 32, 1008 (1980).

104. K. Fujii et al., Nucl. Phys. B 197, 365 (1982).

105. H. Dutz et al., Nucl. Phys. A 601, 319 (1996).

106. K. Egawa et al., Nucl. Phys. B 188, 11 (1981).

107. P.J. Bussey et al., Nucl. Phys. B 169, 403 (1980).

108. A.A. Belyaev et al., Yad. Fiz. 40, 133 (1984).

109. A.A. Belyaev et al., Yad. Fiz. 43, 1469 (1986).

110. E.F. McNicoll et al., Phys. Rev. C 82, 035208 (2010).

111. V. Crede et al., Phys. Rev. C 80, 055202 (2009).

112. O. Bartholomy et al., Eur. Phys. J. A 33, 133 (2007).

113. J. Ajaka et al., Phys. Rev. Lett. 81, 1797 (1998).

114. O. Bartalini et al., Eur. Phys. J. A 33, 169 (2007).

115. D. Elsner et al., Eur. Phys. J. A 33, 147 (2007).

116. M.E. McCracken et al., Phys. Rev. C 81, 025201 (2010).

117. R.G.T. Zegers et al., Phys. Rev. Lett. 91, 092001 (2003).

118. A. Lleres et al., Eur. Phys. J. A 31, 79 (2007).

119. A. Lleres et al., Eur. Phys. J. A 39, 149 (2009).

120. R. Bradford et al., Phys. Rev. C 75, 035205 (2007).

121. B. Dey et al., Phys. Rev. C 82, 025202 (2010).

122. B. Carnahan, Strangeness photoproduction in the $\gamma p \rightarrow$ $K^{0} \Sigma^{+}$reaction, thesis, Catholic University, UMI-310909682 (2003).

123. R. Lawall et al., Eur. Phys. J. A 24, 275 (2005).

124. R. Castelijns et al., Eur. Phys. J. A 35, 39 (2008).

125. R. Ewald et al., AIP Conf. Proc. 1257, 566 (2010).

126. D.M. Manley, R.A. Arndt, Y. Goradia, V.L. Teplitz, Phys. Rev. D 30, 904 (1984).

127. A. Anisovich, E. Klempt, A. Sarantsev, U. Thoma, Eur. Phys. J. A 24, 111 (2005).

128. A.V. Anisovich, A.V. Sarantsev, Eur. Phys. J. A 30, 427 (2006).

129. J. Blatt, V. Weisskopf, Theoretical Nuclear Physics (John \& Sons, New York, 1952).
130. N. Kaiser, P.B. Siegel, W. Weise, Phys. Lett. B 362, 23 (1995).

131. U.-G. Meissner, J.A. Oller, Nucl. Phys. A 673, 311 (2000).

132. J. Nieves, E. Ruiz Arriola, Phys. Rev. D 64, 116008 (2001).

133. M. Doring, C. Hanhart, F. Huang, S. Krewald, U.G. Meissner, Phys. Lett. B 681, 26 (2009).

134. M. Doring, C. Hanhart, F. Huang, S. Krewald, U.G. Meissner, Nucl. Phys. A 829, 170 (2009).

135. A.V. Anisovich, V. Kleber, E. Klempt, V.A. Nikonov, A.V. Sarantsev, U. Thoma, Eur. Phys. J. A 34, 243 (2007).

136. G. Höhler, H. Schopper, Numerical Data and Functional Relationships in Science and Technology. Group I: Nuclear and Particle Physics, Vol. 9: Elastic and Charge Exchange Scattering of Elementary Particles. B: Pion Nucleon Scattering, Pt. 2: Methods and Results, LandoltBörnstein, New Series, I/9B2 (Springer, Berlin, 1983).

137. D.M. Manley, E.M. Saleski, Phys. Rev. D 45, 4002 (1992).

138. T.P. Vrana, S.A. Dytman, T.S.H. Lee, Phys. Rep. 328, 181 (2000).

139. L. Tiator, S.S. Kamalov, S. Ceci, G.Y. Chen, D. Drechsel, A. Svarc, S.N. Yang, Phys. Rev. C 82, 055203 (2010).

140. M. Hadzimehmedovic, S. Ceci, A. Svarc, H. Osmanovic, J. Stahov, Phys. Rev. C 84, 035204 (2011).

141. D.H. Saxon, R.D. Baker, K.W. Bell, J.A. Blissett, I.J. Bloodworth, T.A. Broome, J.C. Hart, A.L. Lintern et al., Nucl. Phys. B 162, 522 (1980).

142. K.W. Bell, J.A. Blissett, T.A. Broome, H.M. Daley, J.C. Hart, A.L. Lintern, R. Maybury, A.G. Parham et al., Nucl. Phys. B 222, 389 (1983).

143. K.H. Glander, J. Barth, W. Braun, J. Hannappel, N. Jopen, F. Klein, E. Klempt, R. Lawall et al., Eur. Phys. J. A 19, 251 (2004).

144. T. Mart, C. Bennhold, Phys. Rev. C 61, 012201 (2000).

145. A.V. Anisovich, A. Sarantsev, O. Bartholomy, E. Klempt, V.A. Nikonov, U. Thoma, Eur. Phys. J. A 25, 427 (2005).

146. A.V. Sarantsev, V.A. Nikonov, A.V. Anisovich, E. Klempt, U. Thoma, Eur. Phys. J. A 25, 441 (2005).

147. R.A. Schumacher, M.M. Sargsian, Phys. Rev. C 83, 025207 (2011). 